\section{Redundant Multi-Mode Filter for a Navigation System}

\author{
DAVID J. ALLERTON \\ University of Sheffield \\ UK \\ HUAMIN JIA \\ Cranfield University \\ UK
}

An approach is introduced to the design of a multi-mode navigation filter to combine a low-cost skewed redundant inertial measurement unit (SRIMU) with a multifunctional GPS (MF-GPS) receiver in order to implement a fault-tolerant aircraft navigation system, which can achieve the required navigation performance of conventional systems in terms of accuracy, integrity, continuity, and availability. The MF-GPS receiver provides raw GPS measurements for pseudo-range and range rate to compute the navigation solutions (position and velocity) and also multi-antenna carrier phase interferometric measurements to estimate the aircraft attitude solution, if the carrier phase data is reliable. A multi-mode navigation filter is designed which combines state and measurement fusion methods and processes the SRIMU and raw MF-GPS outputs to provide reliable position, velocity and attitude information, and also kinematic parameters required in control, guidance, and navigation applications. The feasibility and performance of this integrated design is assessed and evaluated by using simulation. The accuracy of inertial gyros used in the evaluation ranges from $1 \%$ to $30^{\circ} / \mathbf{h}$, including low-cost inertial sensor technologies. The simulation studies presented here show that a multi-mode navigation filter can achieve sufficient reliability and accuracy and that SRIMU/MF-GPS integrated navigation systems may provide a cost-effective system for future regional aircraft, general aviation aircraft, and unmanned aerial vehicles.

Manuscript received November 4, 2005; revised January 23, 2006; released for publication April 15, 2006.

IEEE Log No. T-AES/43/1/895041.

Refereeing of this contribution was handled by G. Lachapelle.

This work was supported by the European Commission under the Framework V programme, through the SHINE (Smart Hybrid Integrated Navigation Equipment) project, Contract G4RD-CT-2000-00227. The support from Thales is gratefully acknowledged.

Authors' addresses: D. J. Allerton, Dept. of Automatic Control and Systems Engineering, University of Sheffield, Mappin St., Sheffield S1 3JD, UK, E-mail: (d.j.allerton@ @heffield.ac.uk); H. Jia, School of Engineering, Cranfield University, Cranfield, Bedford MK43 0AL, UK.

$\overline{0018-9251 / 07 / \$ 25.00 ~(C) ~} 2007$ IEEE

\section{INTRODUCTION}

Approaches to the design and development of fault-tolerant aircraft navigation systems have been studied for over three decades. During this period, many fault-tolerant aircraft navigation system architectures, data fusion filter architectures, and filtering algorithms have been proposed, including centralized, cascaded, federated, and distributed (decentralized) filter architectures and algorithms [1]. A wide range of publications cover the integration of inertial navigation system (INS) and the Global Positioning Systems (GPS) using Kalman filtering techniques $[2,3]$. The design of an integrated navigation filter is either based on the navigation state solutions given by GPS (referred to as state fusion) or relies on raw GPS measurements (including pseudo-range), Doppler, and/or GPS carrier phase (known as measurement fusion). The estimates given by the filter can be used to periodically update the INS-based navigation states (open-loop mechanism) or to constrain inertial sensor errors and correct the navigation state errors (closed-loop mechanism). Recent research has focused on the use of inertial information to aid the GPS receiver carrier and code tracking loops, effectively reducing the bandwidth of these loops to improve the antijamming capability and dynamic performance of a GPS receiver [4]. These closed-loop systems provide significant improvements in estimation of the navigation state in comparison with open-loop systems.

Developments of GPS carrier phase interferometric measurements to determine aircraft attitude have shown that a multi-antenna GPS receiver with innovative GPS attitude determination algorithms can provide measurements of aircraft attitude with acceptable accuracy, subject to GPS signal quality [5-7], and many reliable integer ambiguity resolution techniques have been presented for GPS-based attitude determination [8-10]. Integration of an INS with a GPS receiver incorporating attitude determination can improve both the long-term attitude stability and the short-term attitude accuracy. In these applications, the navigation filter is typically provided by a state fusion Kalman filter, where the differences between the navigation state solutions from an INS and a GPS attitude receiver provide the observables of the filter [11].

Integration of a low-cost attitude heading reference system (AHRS) with a GPS attitude receiver is reported by Gebre-Egziabher [12], where an attitude fusion filter with a constant gain blends the attitude solution from inertial sensors and the attitude solution from a GPS attitude receiver; the angular rates are used to propagate the attitude solutions in time. Other methods use the attitude information from inertial systems and other sensors to aid GPS attitude determination, by reducing the search space to resolve 
integer ambiguity [13, 14]. However, few studies have compared the performance of SRIMU/MF-GPS integrated navigation systems incorporating different qualities of inertial sensors or considered the effects of GPS measurements in the design of a multi-mode navigation filter. In addition, when simple angular rate models and constant filter gains are used to propagate the attitude solution (rather than a more complex inertial error model), high-quality gyros and a faster update rate of the GPS attitude solutions are necessary in order to reduce the attitude propagation error. Although a $10 \mathrm{~Hz}$ attitude output rate has been reported [6], development of a reliable GPS-based attitude determination algorithm is challenging, particularly for cycle slip detection and integer ambiguity resolution in flexible wing structures.

Most current integrated systems are limited to orthogonally-configured inertial measurement units (IMUs) where the inertial measurement sensed by one sensor mounted in one axis is independent of measurements of sensors mounted in the other axes. This configuration offers several advantages in terms of IMU error calibration models and manufacturing. Several IMU error calibration models have been applied to the design of integrated navigation filters to correct orthogonal IMU errors. However, an aircraft navigation system usually needs more than three IMUs to provide the necessary fault tolerance of the navigation functions, with concomitant increases in weight and power consumption.

With recent advances in sensor technologies, low-cost small-size tactical-grade inertial sensors have been developed, which can be integrated in a single suite using nonorthogonal configurations, where the sensing axes of the inertial sensors are skewed with respect to the orthogonal axes of the inertial suite. Such IMUs are usually known as skewed-redundant IMUs (SRIMUs). In comparison with traditional fault-tolerant IMU designs [1], one SRIMU suite can replace multiple IMUs to reduce the cost, size, and mass of the inertial navigation equation, and provide redundant inertial measurements (angular rates and accelerations) using fewer inertial sensors to increase the reliability and fault tolerance of an integrated navigation system. SRIMU designs can exploit the benefits of emerging inertial sensor technologies and high-speed embedded microprocessor systems and will provide the basis for the future generations of fault-tolerant navigation systems. Integration of a low-cost SRIMU with a multifunctional GPS receiver (SRIMU/MF-GPS) offers the potential to reduce the cost, size, and power consumption of aircraft navigation systems, while meeting the required navigation performance (RNP) of aircraft navigation systems in terms of accuracy, integrity, continuity, and availability. However, no significant research has been undertaken to evaluate the accuracy required by inertial sensors integrated with an MF-GPS receiver or the effects of update rate of GPS attitude information.

One major problem with SRIMUs is that conventional IMU error calibration models cannot be used in the design of an SRIMU/MF-GPS integrated navigation filter because the required inertial quantities are a linear combination of all SRIMU measurements and the definition of sensor misalignments in SRIMU configurations is different from that in an orthogonal IMU configuration. In previous SRIMU studies, emphasis has been given to detection and isolation of inertial sensor failures [15, 16]. For example, Hall [16] describes a parity vector compensation method for sensor failure detection and isolation (FDI) where the combined sensor errors are modeled as a first-order Markov process and then estimated by means of a 12-state Kalman filter using the parity residuals as the filter observables. This compensation technique enhances the effect of true failure signals on the parity residual vector and can improve the performance of generalized likelihood ratio test (GLRT) algorithms. However, this error compensation filter cannot correct each physical sensor in an SRIMU configuration owing to the lack of a proper SRIMU error model. Allerton [17] proposes an SRIMU error calibration model which redefines the inertial sensor misalignments and can be used to compensate for SRIMU measurement errors. In addition, the redundant information provided by an SRIMU system can be used for both the detection of sensor failures and also for the correction of the SRIMU measurements in the design of a multi-mode navigation filter by increasing the observability of inertial sensor errors.

This paper describes the design of a multi-mode navigation filter used in the development of SRIMU/MF-GPS integrated navigation systems. The feasibility and performance of this integration design is assessed and evaluated by simulation methods. The accuracy of the inertial gyros used in the evaluation ranges from $1 \% / \mathrm{h}$ to $30^{\circ} / \mathrm{h}$, covering current low-cost inertial sensor technologies used in applications including regional aircraft, general aviation, and unmanned aerial vehicles. The paper is organized as follows: Section II introduces the system architecture. The design of a multi-mode navigation filter is described in Section III. Section IV discusses SRIMU/MF-GPS error dynamic models and the simulation results are presented in Section V.

\section{FUNCTIONAL SYSTEM ARCHITECTURE}

The research originated from the Smart Hybrid Integrated Navigation Equipment (SHINE) project, supported by the EU Framework V programme. In the SHINE system, an SRIMU and a dual-antenna GPS receiver were integrated into a single unit to provide reliable position, velocity, attitude information, and kinematic parameters required by avionics 


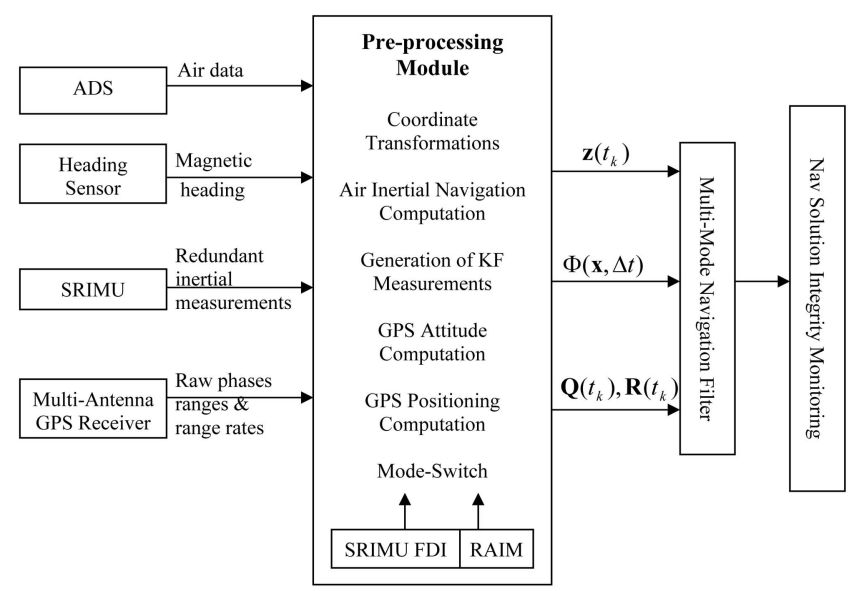

Fig. 1. Functional system architecture.

systems for control, guidance, and navigation. The SRIMU consists of multiple accelerometers and gyros providing redundant inertial measurements. A dual-antenna GPS receiver is used with an antenna baseline of approximately $2 \mathrm{~m}$ to estimate the aircraft pitch and heading. The research reported here extends this approach to SRIMU/MF-GPS integration. The dual-antenna GPS receiver is replaced by a multifunctional GPS receiver, which provides the GPS-based navigation states and raw GPS measurements.

Although many innovative GPS-based attitude determination algorithms have been proposed, this paper focuses on the design of multi-mode navigation filter algorithms rather than GPS-based attitude-determination algorithms. The functional architecture of the overall integrated system is shown in Fig. 1 where $\mathbf{z}\left(t_{k}\right)$ is the normalized measurement vector, $\mathbf{\Phi}(\mathbf{x}, \Delta \mathbf{t})$ is the adjustable system transition matrix, and $\mathbf{Q}\left(t_{k}\right)$ and $\mathbf{R}\left(t_{k}\right)$ are the covariances of the system and measurement noise, respectively. All matrices are generated by the preprocessing module. Air data sensors (ADS), which provide air speed and barometric altitude, and a magnetic heading sensor are integrated with the SRIMU/MF-GPS system to improve the observability of the multi-mode navigation filter.

In this architecture, the SRIMU FDI detects inertial sensor failures whereas RAIM (receiver autonomous integrity monitoring) is used for auxiliary GPS FDI [18-20]. The SRIMU FDI algorithm extends the basic GLRT algorithm $[15,16]$ by adding an SRIMU compensation filter and a sequential GLRT algorithm to improve the performance of the GLRT algorithm, particularly the capability to detect mid-value and drift failures. Both the SRIMU FDI and RAIM use redundant measurements to detect sensor signal failures. With the exception of the initialization of data for the multi-mode navigation filter, the preprocessor performs the GPS-based attitude, positioning and inertial navigation computations derived from GPS measurements to provide backup functions for the multi-mode navigation filter. The multi-mode navigation filter estimates the navigation state and sensor errors and also compensates for the sensor measurement errors. The integrity of the navigation solutions given by the navigation filter is monitored at the system level by the navigation solution integrity monitoring (NSIM) function, which is based on covariance analysis and a statistical test of the filter residuals [21-23]. This paper focuses on the design of a multi-mode navigation filter; the design of the SRIMU compensation filter and sequential FDI algorithms is outside the scope of the paper [26].

\section{MULTI-MODE NAVIGATION FILTER}

\section{A. Kalman Filter Algorithm}

The multi-mode navigation filter developed here is based on the Kalman filtering techniques. Consider the discrete-time stochastic process, where the system and measurement models are given by

$$
\begin{aligned}
& \mathbf{x}\left(t_{k}\right)=\boldsymbol{\Phi}\left(t_{k}, t_{k-1}\right) \mathbf{x}\left(t_{k-1}\right)+\mathbf{G}\left(t_{k-1}\right) \boldsymbol{v}\left(t_{k-1}\right) \\
& \mathbf{z}\left(t_{k}\right)=\mathbf{H}\left(t_{k}\right) \mathbf{x}\left(t_{k}\right)+\mathbf{w}\left(t_{k}\right)
\end{aligned}
$$

where $\mathbf{x}$ is an $n$-system state, $\Phi$ is an $n \times n$ state transition matrix, $\mathbf{H}$ is an $m \times n$ measurement matrix, $\mathbf{G}$ is an $n \times q$ matrix, $\mathbf{z}$ is an $m$-measurement vector, $\boldsymbol{v}$ is an $q$-additive process noise which takes account of the system perturbations, and $\mathbf{w}$ is an $m$-additive measurement noise. It is assumed that $\boldsymbol{v}$ and $\mathbf{w}$ are independent, zero-mean, white Gaussian sequences with covariances $\mathbf{Q}$ and $\mathbf{R}$, respectively. The initial system state $\mathbf{x}\left(t_{0}\right)$ is a Gaussian distributed random variable with an initial value $\mathbf{x}_{0}$ and covariance $\mathbf{P}_{0}$ and is independent of the noise.

The Kalman filter algorithm implementation is as follows [24].

Step 1 Initialization

$$
\mathbf{P}\left(t_{0}\right)=\mathbf{P}_{0}, \quad \hat{\mathbf{x}}\left(t_{0}\right)=\mathbf{x}_{0} .
$$

Step 2 Time update (predictor, effect of dynamics)

$$
\begin{aligned}
\hat{\mathbf{x}}\left(t_{k}^{-}\right)= & \boldsymbol{\Phi}\left(t_{k}, t_{k-1}\right) \hat{\mathbf{x}}\left(t_{k-1}^{+}\right) \\
\mathbf{P}\left(t_{k}^{-}\right)= & \boldsymbol{\Phi}\left(t_{k}, t_{k-1}\right) \mathbf{P}\left(t_{k-1}^{+}\right) \Phi^{\mathrm{T}}\left(t_{k}, t_{k-1}\right) \\
& +\mathbf{G}\left(t_{k-1}\right) \mathbf{Q}\left(t_{k-1}\right) \mathbf{G}^{\mathrm{T}}\left(t_{k-1}\right) \\
\mathbf{r}\left(t_{k}\right)= & \mathbf{z}\left(t_{k}\right)-\mathbf{H}\left(t_{k}\right) \hat{\mathbf{x}}\left(t_{k}^{-}\right) \\
\mathbf{S}\left(t_{k}\right)= & \mathbf{H}\left(t_{k}\right) \mathbf{P}\left(t_{k}^{-}\right) \mathbf{H}^{\mathrm{T}}\left(t_{k}\right)+\mathbf{R}\left(t_{k}\right) .
\end{aligned}
$$

Step 3 Measurement update (estimator, effect of measurement)

$$
\begin{aligned}
\mathbf{K}\left(t_{k}\right) & =\mathbf{P}\left(t_{k}^{-}\right) \mathbf{H}^{\mathrm{T}}\left(t_{k}\right) \mathbf{S}^{-1}\left(t_{k}\right) \\
\hat{\mathbf{x}}\left(t_{k}^{+}\right) & =\hat{\mathbf{x}}\left(t_{k}^{-}\right)+\mathbf{K}\left(t_{k}\right) \mathbf{r}\left(t_{k}\right) \\
\mathbf{P}\left(t_{k}^{+}\right) & =\mathbf{P}\left(t_{k}^{-}\right)-\mathbf{K}\left(t_{k}\right) \mathbf{H}\left(t_{k}\right) \mathbf{P}\left(t_{k}^{-}\right) .
\end{aligned}
$$




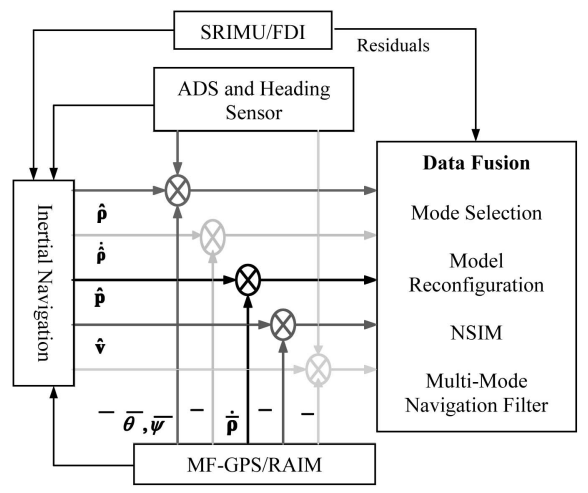

\begin{tabular}{|l|l|}
\hline$\hat{\phi}, \hat{\theta}, \hat{\psi}$ are the roll, pitch and yaw attitudes from the INS & $\bar{\phi}, \bar{\theta}, \bar{\psi}$ are the roll, pitch and yaw attitudes from the MF-GPS \\
\hline$\hat{\mathbf{p}}, \hat{\mathbf{v}}$ are the position and velocity vectors from the INS & $\overline{\mathbf{p}}, \overline{\mathbf{v}}$ are the position and velocity vectors from the MF-GPS \\
\hline $\begin{array}{l}\hat{\mathbf{\rho}}, \dot{\hat{\mathbf{\rho}}} \text { are the range and range rate vectors estimated by the } \\
\text { INS }\end{array}$ & $\begin{array}{l}\overline{\mathbf{\rho}}, \dot{\overline{\mathbf{\rho}}} \text { are the range and range rate vectors measured by the MF- } \\
\text { GPS }\end{array}$ \\
\hline
\end{tabular}

Fig. 2. Multi-mode filter architecture.

TABLE I

Operating Modes

\begin{tabular}{cccccc}
\hline \hline SRIMU & ADS & $\begin{array}{c}\text { Heading } \\
\text { Sensor }\end{array}$ & $\begin{array}{c}\text { GPS } \\
\text { Observations }\end{array}$ & $\begin{array}{c}\text { GPS-Based } \\
\text { Attitude }\end{array}$ \\
\hline X & $\mathrm{X}$ & $\mathrm{X}$ & $\mathrm{X}$ & $\mathrm{X}$ & KF Mode \\
$\mathrm{X}$ & $\mathrm{X}$ & $\mathrm{X}$ & $\mathrm{X}$ & & Mode-F (all measurements available) \\
$\mathrm{X}$ & & $\mathrm{X}$ & $\mathrm{X}$ & $\mathrm{X}$ & $\begin{array}{c}\text { Mode-GPSAtt (lost GPS attitude determination) } \\
\text { Mode-H (lost magnetic heading sensor) } \\
\text { X }\end{array}$ \\
$\mathrm{X}$ & $\mathrm{X}$ & $\mathrm{X}$ & $\mathrm{X}$ & $\mathrm{X}$ & Mode-AH (lost ADS and heading sensor) \\
Mode-SRIMU (GPS outage)
\end{tabular}

By examining the predictor and estimator given above, the filter algorithm provides useful statistical information that can be used to monitor both the convergence and the consistency of the filter estimation procedure. The outputs of the filter predictor include the filter innovation $\mathbf{r}\left(t_{k}\right)$ and its covariance $\mathbf{S}\left(t_{k}\right)$, while the filter estimator outputs the filter residual $\mathbf{r}\left(t_{k}^{+}\right)$and the residual covariance $\mathbf{S}\left(t_{k}^{+}\right)$, defined as follows:

$$
\begin{aligned}
\mathbf{r}\left(t_{k}^{+}\right) & =\mathbf{z}\left(t_{k}\right)-\mathbf{H}\left(t_{k}\right) \mathbf{x}\left(t_{k}^{+}\right) \\
\mathbf{S}\left(t_{k}^{+}\right) & =\mathbf{H}\left(t_{k}\right) \mathbf{P}\left(t_{k}^{+}\right) \mathbf{H}^{\mathrm{T}}\left(t_{k}\right)+\mathbf{R}\left(t_{k}\right) .
\end{aligned}
$$

It has been shown that the filter residual process is a zero-mean white Gaussian random sequence in normal operations, if the Kalman filter model matches the true system model [22-24]. This feature is usually exploited in the analysis of Kalman filter integrity for failure detection and also for checking the consistency of measurement data and monitoring the filter divergence.

\section{B. Multi-Mode Navigation Filter Architecture}

The proposed multi-mode navigation filter architecture is shown in Fig. 2 and the operating modes of the navigation filter are summarized in Table I, where the modes are selected on the basis of available measurement information. The SRIMU/FDI module generates estimates of the specific force triad, the angular rate triad and the sensor health status report, which are used to reconfigure the inertial system and the multi-mode navigation filter. It also outputs the SRIMU measurement residuals, which are used as the observables of the multi-mode navigation filter. The ADS and heading sensor module provide pressure altitude compensation needed for the stabilization of the vertical channel of the inertial system, as well as heading and air speed measurements for the multi-mode navigation filter. The inertial navigation module combines the SRIMU/FDI module outputs, pressure altitude, and the positions of visible GPS satellites from the MF-GPS module to provide coarse estimates of the navigation states and the computed range and range rate of the visible GPS satellites. One additional function of this module is to provide backup for the multi-mode navigation filter when GPS signals are unavailable. The architecture of the inertial module, including other functions implemented in the module, is illustrated in Fig. 3. The MF-GPS/RAIM module outputs GPS-derived navigation states and corrected pseudo-range and range rates. The MF-GPS outputs are also used in the NSIM module.

The data fusion method is based on an extended Kalman filter algorithm, where all filter states are 


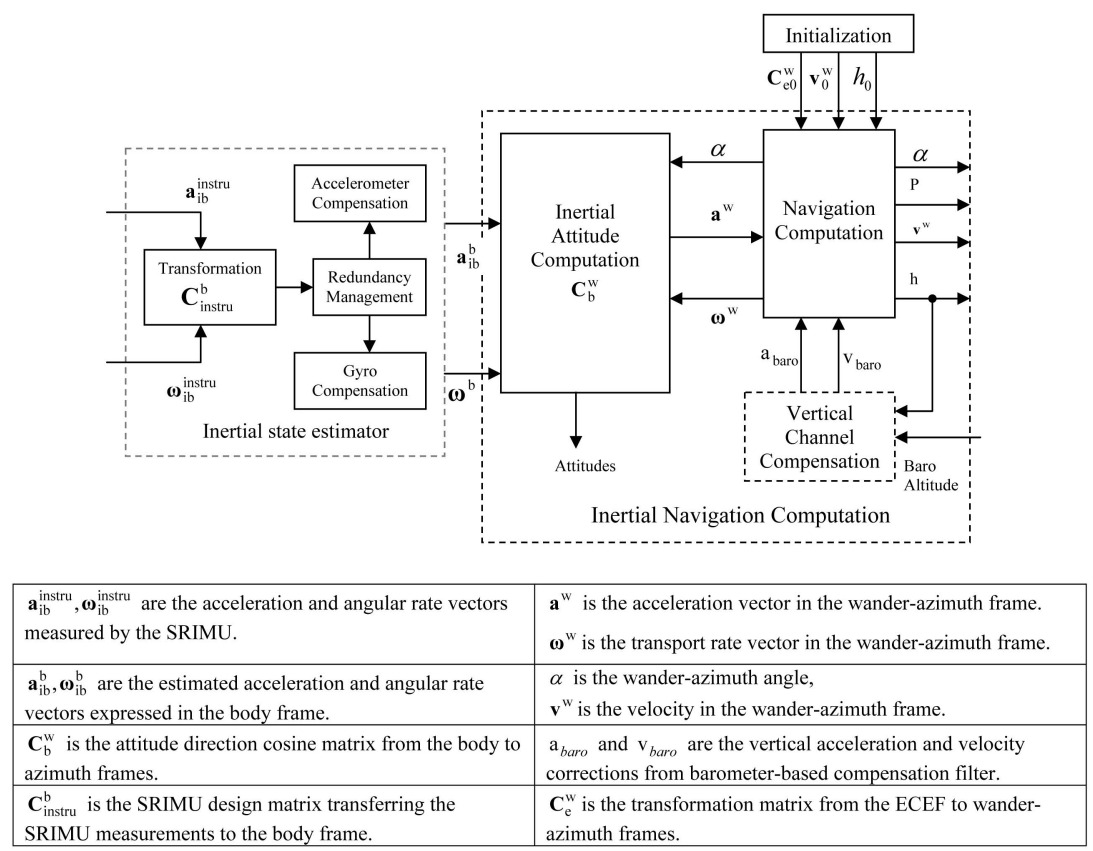

Fig. 3. Inertial navigation computation.

error states. The functions performed by the data fusion module are as follows.

1) Process the raw sensor measurements to generate normalized data for the multi-mode navigation filter.

2) Determine the operating mode according to the sensor health status reports from the FDI and RAIM modules.

3) Reconfigure the dynamic model and the measurement model of the multi-mode navigation filter.

4) Perform the Kalman filter functions to correct the coarse estimates of the navigation states.

5) Correct the SRIMU and other sensor errors.

6) Monitor any abnormality of the state estimates of the navigation filter.

The filter state vector is subdivided into two subvectors, the basic error state vector and the sensor error state vector as follows:

$$
\begin{aligned}
\mathbf{x} & =\left[\begin{array}{c}
\mathbf{x}_{\text {basic state errors }} \\
\mathbf{x}_{\text {sensor errors }}
\end{array}\right] \\
\mathbf{x}_{\text {basic state errors }}= & {\left[\begin{array}{l}
\mathrm{x}_{1} \\
\mathrm{x}_{2} \\
\mathrm{x}_{3} \\
\mathrm{x}_{4} \\
\mathrm{x}_{5} \\
\mathrm{x}_{6} \\
\mathrm{x}_{7} \\
\mathrm{x}_{8} \\
\mathrm{x}_{9}
\end{array}\right]=\left[\begin{array}{c}
\delta \varphi_{\mathrm{R}} \\
\delta \lambda_{\mathrm{R}} \\
\delta \mathrm{h} \\
\delta \mathrm{v}_{\mathrm{x}}^{1} \\
\delta \mathrm{v}_{\mathrm{y}}^{1} \\
\delta \mathrm{v}_{\mathrm{z}}^{1} \\
\partial \phi_{\mathrm{x}} \\
\partial \phi_{\mathrm{y}} \\
\partial \phi_{\mathrm{z}}
\end{array}\right] }
\end{aligned}
$$

$$
\begin{aligned}
\mathbf{x}_{\text {sensor errors }} & =\left[\begin{array}{c}
\text { Rx Clk error etates } \\
\text { SRIMU accel error states } \\
\text { SRIMU gyro error states } \\
\text { magnetic heading error state } \\
\text { barometer bias state } \\
\text { ADS air speed bias state }
\end{array}\right] \\
= & {\left[\begin{array}{c}
\mathbf{x}_{\text {clk-pha }} \\
\mathbf{x}_{\text {clk-rate }} \\
\mathbf{x}_{\text {accel-1 }} \\
\vdots \\
\mathbf{x}_{\text {accel- } n} \\
\mathbf{x}_{\mathrm{gyro}-1} \\
\vdots \\
\mathbf{x}_{\text {gyro- } n} \\
\mathbf{x}_{\text {Mag }} \\
\mathbf{x}_{\text {Bar }} \\
\mathbf{x}_{\mathrm{ADS}}
\end{array}\right] }
\end{aligned}
$$

The basic error state vector is the navigation state error vector and has a fixed form, but the sensor error state vector depends on the availability of sensors. The SRIMU accelerometer and gyro error vectors are reconfigurable because they are dependent on the SRIMU design matrix, as shown in the following section. The other sensor error states include the barometer measurement bias state, the computed air speed bias state from the ADS and the magnetic heading measurement bias state, which depend on the availability of these sensors. In summary, the dynamic model of the multi-mode navigation filter can be 
formulated as follows:

$$
\begin{aligned}
{\left[\begin{array}{c}
\mathbf{x}_{\text {basic error states }}\left(t_{k}\right) \\
\mathbf{x}_{\text {SRIMU errors }}\left(t_{k}\right) \\
\mathbf{x}_{\text {other sensor errors }}\left(t_{k}\right)
\end{array}\right]=} & {\left[\begin{array}{ccc}
\Phi_{\text {basic }} & \boldsymbol{\Phi}_{\text {RSIMU-Re }} & \Phi_{\text {others-Re }} \\
0 & \boldsymbol{\Phi}_{\text {SRIMU }} & 0 \\
0 & 0 & \Phi_{\text {others }}
\end{array}\right] } \\
& \times\left[\begin{array}{c}
\mathbf{x}_{\text {basic error states }}\left(t_{k-1}\right) \\
\mathbf{x}_{\text {SRIMU errors }}\left(t_{k-1}\right) \\
\mathbf{x}_{\text {other sensor errors }}\left(t_{k-1}\right)
\end{array}\right] \\
& +\mathbf{G}\left(t_{k-1}\right) \boldsymbol{v}\left(t_{k-1}\right) .
\end{aligned}
$$

The measurement vector is given by

$$
\mathbf{z}=\left[\begin{array}{c}
\text { GPS range vector } \\
\text { GPS range rate vector } \\
\text { GPS attitude vector } \\
\text { magnetic heading } \\
\text { air speed } \\
\text { SRIMU residual vector }
\end{array}\right]=\left[\begin{array}{c}
\Delta \mathrm{PR} \\
\Delta \mathrm{PRR} \\
\Delta \mathrm{Att} \\
\Delta \Psi_{\mathrm{M}} \\
\Delta \mathrm{V}_{\mathrm{TAS}} \\
\text { Residuals }
\end{array}\right]
$$

$$
\mathbf{z}\left(t_{k}\right)=\mathbf{D}\left(t_{k}\right) \mathbf{x}\left(t_{k}\right)+\mathbf{w}\left(t_{k}\right) .
$$

The measurement equations of the multi-mode navigation filter vary with the availability of the sensor measurements. The system and measurement models can be dynamically reconfigured according to sensor health reports given by the SRIMU FDI and RAIM which use redundant measurements and the filter residuals to detect sensor signal failures. If the SRIMU FDI detects an inertial sensor failure, the multi-mode navigation filter will dynamically reconfigure the measurement and state transition submatrices, with the exception of the basic state error transition submatrix $\Phi_{\text {basic }}$.

\section{DYNAMIC MODELS OF MULTI-MODE NAVIGATION FILTER}

\section{A. Inertial System Error Dynamic Model}

In this paper, the navigation frame is based on the wander-azimuth frame and the error dynamic models of the inertial system are developed using a perturbation model with respect to the true wander-azimuth frame. The normalized error dynamic models [26] are derived as follows.

1) Velocity Error Differential Equation: The velocity error states are selected in order to simplify the formulation of the error models [25] and are defined in the wander-azimuth frame as follows.

$$
\begin{aligned}
\delta \dot{\mathbf{v}}^{1}= & -\left[\left(\boldsymbol{\omega}_{\mathrm{e} / \mathrm{w}}^{\mathrm{w}} \times\right)+2\left(\boldsymbol{\omega}_{\mathrm{l} / \mathrm{e}}^{\mathrm{w}} \times\right)\right] \delta \mathbf{v}^{1}+\mathbf{f}^{\mathrm{w}} \times \partial \phi+\delta \mathbf{g}^{\mathrm{w}} \\
& -\left(\mathbf{f}^{\mathrm{w}}+\mathbf{g}^{\mathrm{w}}\right) \times \partial \boldsymbol{\theta}+\nabla^{\mathrm{w}}
\end{aligned}
$$

where $\delta \mathbf{v}^{1}$ is the alternative velocity error vector and is defined by $\tilde{\mathbf{v}}^{\mathrm{w}}=[\mathbf{I}-(\partial \theta \times)] \mathbf{v}^{\mathrm{w}}+\delta \mathbf{v}^{1}, \tilde{\mathbf{v}}^{\mathrm{w}}$ and $\mathbf{v}^{\mathrm{w}}$ are the estimated and true velocity vectors, $\mathbf{f}^{\mathrm{w}}$ is the specific force vector, $\mathbf{g}^{\mathrm{w}}$ is the gravity vector, $\omega_{\mathrm{e} / \mathrm{w}}^{\mathrm{w}}$ is the transport rate vector and $\omega_{\mathrm{I} / \mathrm{e}}^{\mathrm{w}}$ is the Earth rate vector, $\partial \boldsymbol{\theta}$ is the angular position error vector, $\partial \phi$ is the phi-angle error vector given in (16), $\delta \mathbf{g}^{\mathrm{w}}$ is the variation of the gravity vector, and $\nabla^{\mathrm{w}}$ is the total accelerometer error vector. The terms $\delta \mathbf{g}^{\mathrm{W}}$ and $\left(\mathbf{f}^{\mathrm{w}}+\mathbf{g}^{\mathrm{w}}\right) \times \partial \boldsymbol{\theta}$ are given by (A.1) and (A.2) in the appendix.

2) Phi-Angle Error Differential Equation: The representation of the phi-angle (bank angle) errors has certain advantages for the correction of the navigation state errors because the estimated errors of the navigation states are directly related to the true navigation frame

$\partial \dot{\phi}=-\left[\left(\omega_{\mathrm{I} / \mathrm{e}}^{\mathrm{w}} \times\right)+\left(\omega_{\mathrm{e} / \mathrm{w}}^{\mathrm{w}} \times\right)\right] \partial \phi+\delta \boldsymbol{\omega}_{\mathrm{e} / \mathrm{w}}^{\mathrm{w}}+\left(\boldsymbol{\omega}_{\mathrm{I} / \mathrm{e}}^{\mathrm{w}} \times\right) \partial \theta-\Delta^{\mathrm{w}}$

where $\Delta^{\mathrm{w}}$ is the total gyro error vector, $\delta \boldsymbol{\omega}_{\mathrm{e} / \mathrm{w}}^{\mathrm{w}}$ is the transport rate error vector given by (35) and the error term $\left.\left(\omega_{\mathrm{e} / \mathrm{w}}^{\mathrm{w}} \times\right)\right] \partial \phi$ is given by (36) in the appendix.

Because the inertial attitude algorithm directly computes the Euler angles, the phi-angle error has to be transferred into the attitude error for the attitude error correction by

$$
\left[\begin{array}{c}
\delta \phi \\
\delta \theta \\
\delta \psi_{\mathrm{w}}
\end{array}\right]=\mathbf{T}_{\text {tilt }}^{\text {Euler_err }}\left[\begin{array}{l}
\partial \phi_{\mathrm{x}} \\
\partial \phi_{\mathrm{y}} \\
\partial \phi_{\mathrm{z}}
\end{array}\right]
$$

where $\mathbf{T}_{\text {tilt }}^{\text {Eul_err }}$ is the transformation matrix between the wander-azimuth attitude error and the phi-angle error and is given by (A.5) in the appendix, $\phi$ is the roll angle, $\delta \phi$ is the roll angle error, $\theta$ is the pitch angle, $\delta \theta$ is the pitch error, $\psi_{\mathrm{w}}$ is the wander heading angle, and $\delta \psi_{\mathrm{w}}$ is the wander heading error.

3) Linear Position Error Differential Equation: The position errors are defined as the linear distance errors in the north-east-up (NEU) frame and are formulated as follows:

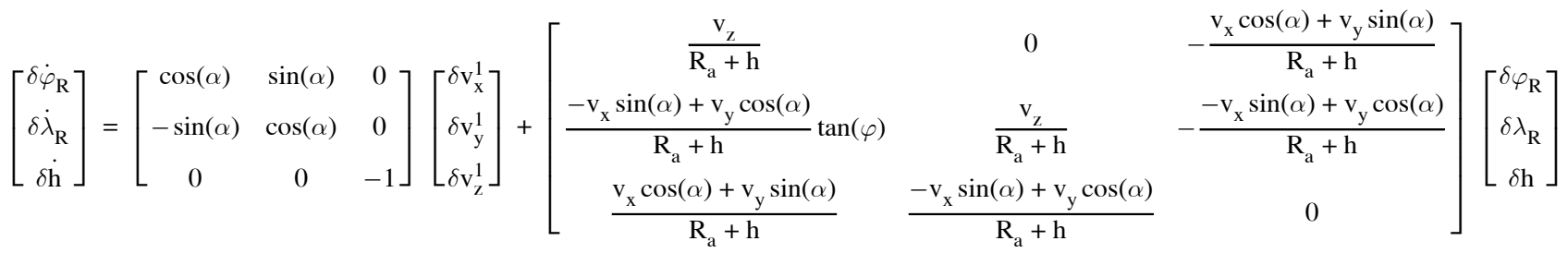


where $\delta \varphi_{\mathrm{R}}=\left(\mathrm{R}_{\mathrm{a}}+\mathrm{h}\right) \delta \varphi$ is the northern position error and $\delta \lambda_{\mathrm{R}}=\left(\mathrm{R}_{\mathrm{a}}+\mathrm{h}\right) \cos (\varphi) \delta \lambda$ is the eastern position error, $\alpha$ is the wander-azimuth angle, $\varphi$ is the latitude, $\lambda$ is the longitude, $\delta \mathrm{h}$ is the height error, and $\mathbf{R}_{\mathrm{a}}$ is the equatorial radius of the Earth. scale factor errors), and $\mathbf{G}$ is an $n \times n$-dimensional matrix, containing sensor misalignments.

The sensor misalignments can lead to a small change of the SRIMU design matrix $\Delta \mathbf{H}_{\text {SRIMU }}$, which is defined as follows:

$$
\begin{aligned}
\Delta \mathbf{H}_{\mathrm{SRIMU}}=\left[\begin{array}{ccc}
-\delta_{\mathrm{El}^{1}} \sin \mathrm{El}_{0}^{1} \cos \mathrm{Az}_{0}^{1} & -\delta_{\mathrm{El}^{1}} \sin \mathrm{El}_{0}^{1} \sin \mathrm{Az}_{0}^{1} & \\
+\delta_{\mathrm{Az}^{1}} \cos \mathrm{El}_{0}^{1} \sin \mathrm{Az}_{0}^{1} & -\delta_{\mathrm{Az}^{1}} \cos \mathrm{El}_{0}^{1} \cos \mathrm{Az}_{0}^{1} & \delta_{\mathrm{El}^{1}} \cos \mathrm{El}_{0}^{1} \\
-\delta_{\mathrm{El}^{2}} \sin \mathrm{El}_{0}^{2} \cos \mathrm{Az}_{0}^{2} & -\delta_{\mathrm{El}^{2}} \sin \mathrm{El}_{0}^{2} \sin \mathrm{Az}_{0}^{2} & \\
+\delta_{\mathrm{Az}^{2}} \cos \mathrm{El}_{0}^{2} \sin \mathrm{Az}_{0}^{2} & -\delta_{\mathrm{Az}^{2}} \cos \mathrm{El}_{0}^{2} \cos \mathrm{Az}_{0}^{2} & \delta_{\mathrm{El}^{2}} \cos \mathrm{El}_{0}^{2} \\
\vdots & \vdots & \vdots \\
-\delta_{\mathrm{El}^{n}} \sin \mathrm{El}_{0}^{n} \cos \mathrm{Az}_{0}^{n} & -\delta_{\mathrm{El}^{n}} \sin \mathrm{El}_{0}^{n} \sin \mathrm{Az}_{0}^{n} & \\
+\delta_{\mathrm{Az}^{n}} \cos \mathrm{El}_{0}^{n} \sin \mathrm{Az}_{0}^{n} & -\delta_{\mathrm{Az}^{n}} \cos \mathrm{El}_{0}^{n} \cos \mathrm{Az}_{0}^{n} & \delta_{\mathrm{El}^{n}} \cos \mathrm{El}_{0}^{n}
\end{array}\right] \quad \text { or } \\
\Delta \mathbf{H}_{\mathrm{SRIMU}}=\operatorname{Diag}\left(\delta_{\mathrm{El}}\right) \Gamma+\operatorname{Diag}\left(\delta_{\mathrm{Az}}\right) \Theta
\end{aligned}
$$

As (18) is used to estimate the linear position error in the NEU frame and (15) and (16) need the angular position error vector, the linear position error has to be transferred into the angular position error by

$$
\left[\begin{array}{l}
\delta \varphi \\
\delta \lambda \\
\delta \alpha
\end{array}\right]=\mathbf{T}_{\text {ang_err }}^{\text {geoerr }}\left[\begin{array}{l}
\partial \theta_{\mathrm{x}} \\
\partial \theta_{\mathrm{y}} \\
\partial \theta_{\mathrm{z}}
\end{array}\right]
$$

where $\mathbf{T}_{\text {ang_err }}^{\text {geo_err }}$ is the transformation matrix between the linear position error and the angular position error and is given by (38) in the appendix.

4) SRIMU Sensor Error Model: The SRIMU measurement equation in [17] is modeled as follows:

$$
\begin{aligned}
{\left[\begin{array}{c}
m_{1} \\
m_{2} \\
\vdots \\
m_{n}
\end{array}\right] } & =\left[\begin{array}{ccc}
\mathbf{i} \circ \mathbf{s}^{1} & \mathbf{j} \circ \mathbf{s}^{1} & \mathbf{k} \circ \mathbf{s}^{1} \\
\mathbf{i} \circ \mathbf{s}^{2} & \mathbf{j} \circ \mathbf{s}^{2} & \mathbf{k} \circ \mathbf{s}^{2} \\
\vdots & \vdots & \vdots \\
\mathbf{i} \circ \mathbf{s}^{n} & \mathbf{j} \circ \mathbf{s}^{n} & \mathbf{k} \circ \mathbf{s}^{n}
\end{array}\right]\left[\begin{array}{c}
\omega_{\mathrm{x}} \\
\omega_{\mathrm{y}} \\
\omega_{\mathrm{z}}
\end{array}\right]+\left[\begin{array}{c}
v_{1} \\
v_{2} \\
\vdots \\
v_{n}
\end{array}\right] \\
\mathbf{m} & =\mathbf{H}_{\text {SRIMU }} \boldsymbol{\omega}+\boldsymbol{v}
\end{aligned}
$$

where the symbols $\mathbf{i}, \mathbf{j}$, and $\mathbf{k}$ are three orthogonal unit vectors, defining an orthogonal frame; the symbol $\circ$ represents the dot product of two vectors; $\mathbf{s}^{i}$ is a unit vector along the sensing direction of sensor $i$ (defined in terms of the azimuth and elevation angles in the orthogonal frame) and $\mathbf{H}_{\text {SRIMU }}$ is the SRIMU design matrix.

To simplify the equations, the compensated SRIMU measurement model is described by

$$
\mathbf{m}=\mathbf{H}_{\mathrm{SRIMU}} \boldsymbol{\omega}+\delta_{\mathrm{D}}+\mathbf{S}_{\mathrm{SF}} \mathbf{m}+\mathbf{G m}+\boldsymbol{v}
$$

where $\delta_{\mathrm{D}}$ is an $n$-dimensional sensor drift error vector, $\mathbf{S}_{\mathrm{SF}}$ is an $n \times n$-dimensional diagonal matrix (defining where $\mathrm{El}^{i}$ and $\mathrm{Az}^{i}$ are the installation elevation and azimuth angles of sensor $i$.

The total error vector of the gyro sensors in the SRIMU can be modeled as follows:

$$
\begin{aligned}
\Delta^{\mathrm{w}}= & \mathbf{C}_{\mathrm{b}}^{\mathrm{w}} \mathbf{C}_{\text {instru }}^{\mathrm{b}} \delta_{\mathrm{D}}+\mathbf{C}_{\mathrm{b}}^{\mathrm{w}} \mathbf{C}_{\text {instru }}^{\mathrm{b}} \operatorname{Diag}(\mathbf{m}) \delta_{\mathrm{SF}} \\
& +\mathbf{C}_{\mathrm{b}}^{\mathrm{w}} \mathbf{C}_{\text {instru }}^{\mathrm{b}} \operatorname{Diag}(\Pi) \delta_{\mathrm{Az}}+\mathbf{C}_{\mathrm{b}}^{\mathrm{w}} \mathbf{C}_{\text {instru }}^{\mathrm{b}} \operatorname{Diag}(\Sigma) \delta_{\mathrm{El}}
\end{aligned}
$$

where $\Pi=\Theta \mathbf{C}_{\text {instru }}^{\mathrm{b}} \mathbf{m}$ and $\Sigma=\Gamma \mathbf{C}_{\mathrm{instru}}^{\mathrm{b}} \mathbf{m}, \delta_{\mathrm{Az}}$ is the azimuth misalignment angle vector of the gyro installations, $\delta_{\mathrm{El}}$ is the elevation misalignment angle vector of the gyro installations, and $\delta_{\mathrm{SF}}$ is an $n$-dimensional scale factor error vector. Diag() denotes a diagonal matrix, the elements of the matrix $\Gamma$ correspond to the coefficients of the elevation misalignments in $\Delta \mathbf{H}_{\text {SRIMU }}$ and the elements of $\Theta$ are the coefficients of azimuth misalignments in $\Delta \mathbf{H}_{\text {SRIMU }}$.

The dynamics of the error terms occurring in (21) are modeled by a combination of a random constant, random walk and exponentially correlated random processes. Depending on the characteristics of the inertial sensor error sources [27], the SRIMU drift error is modeled by a combination of a random constant process, a random walk process and a first-order Gauss-Markov process. The scale factor error, occurring as a result of temperature sensitivity or drift, is modeled as a first-order Gauss-Markov process and all misalignment errors are modeled as random constant processes. The parameters for modeling these stochastic processes are given in Table II. To model actual inertial sensors, these parameters would be obtained from the sensor data sheet. The total accelerometer error vector in the SRIMU has the same form as (21). 
TABLE II

Simulation Parameters of Inertial Sensors

\begin{tabular}{|c|c|c|c|c|c|}
\hline \multirow[b]{2}{*}{ Parameters } & \multicolumn{5}{|c|}{ Sensor } \\
\hline & 1 & 2 & 3 & 4 & 5 \\
\hline Gyro drift time const (sec) & 480 & 470 & 465 & 475.5 & 590 \\
\hline Gyro drift err $(\mathrm{deg} / \mathrm{h})$ & 0.74 & 0.7 & 0.69 & 0.71 & 0.65 \\
\hline Gyro bias err $(\mathrm{deg} / \mathrm{h})^{*}$ & $30|20| 10 \mid 1$ & $30|20| 10 \mid 1$ & $30|20| 10 \mid 1$ & $30|20| 10 \mid 1$ & $30|20| 10 \mid 1$ \\
\hline Gyro SF err time const (sec) & 280 & 260 & 270 & 260 & 300 \\
\hline Gyro SF error (ppm) & 50 & 50 & 50 & 50 & 50 \\
\hline Gyro Az misalign err (arcsec) & 43 & 37 & 38 & 37 & 37 \\
\hline Gyro El misalign err (arcsec) & 44 & 38 & 38 & 40 & 36 \\
\hline Gyro noise (deg/sqrt(hr)) & 0.71 & 0.70 & 0.78 & 0.72 & 0.68 \\
\hline Accel drift time const (sec) & 360 & 360 & 365 & 360.5 & 366 \\
\hline Accel drift err (ug) & 160 & 170 & 154 & 165 & 175 \\
\hline Accel bias err (ug) & 310 & 300 & 300 & 300 & 312 \\
\hline Accel SF err time const (sec) & 250 & 260 & 250 & 260 & 245 \\
\hline Accel SF err (ppm) & 400 & 415 & 420 & 410 & 420 \\
\hline Accel Az misalign err (arcsec) & 45 & 48 & 47 & 48 & 41 \\
\hline Accel El misalign err (arcsec) & 46 & 47 & 45 & 42 & 40 \\
\hline Accel noise (ug/sqrt(hz) & 100 & 100 & 105 & 100 & 102 \\
\hline
\end{tabular}

\section{B. Normalized Filter Observables}

The navigation state errors are represented in the navigation frame whereas the GPS-based navigation state error models apply to the Earth-centered Earth-fixed (ECEF) frame. To develop a multi-mode filter, it is necessary to represent the states in both models in a unified reference coordinate system and the NEU frame was selected as the preferred reference frame.

1) Normalized GPS Pseudo-Range and Range Rate Measurement Equations

The GPS pseudo-range and range rate measurement equations are nonlinear algebraic equations of the navigation states and can be linearized as follows [26]:

$$
\begin{aligned}
& \delta \mathbf{r}=\mathbf{H}_{\mathrm{GNSS}} \delta \mathbf{p}_{\mathrm{p}}+\boldsymbol{v}_{\mathrm{r}} \\
& \delta \dot{\mathbf{r}}=\mathbf{H}_{\mathrm{GNSS}} \delta \dot{\mathbf{p}}_{\mathrm{p}}+\mathbf{H}_{\mathrm{LOSV}} \delta \mathbf{p}+\boldsymbol{v}_{\dot{\mathrm{r}}}
\end{aligned}
$$

where $\delta \mathbf{r}$ is the range residual vector and $\delta \dot{\mathbf{r}}$ is the range rate residual vector, $\mathbf{H}_{\mathrm{GNSS}}$ and $\mathbf{H}_{\mathrm{LOSV}}$ are given by (39) and (40) in the appendix, $\delta \mathbf{p}=\left[\begin{array}{lll}\delta \mathrm{x} & \delta \mathrm{y} & \delta \mathrm{z}\end{array}\right]^{\mathrm{T}}$ is the position error in the ECEF frame, $\delta \mathbf{p}_{\mathrm{p}}=$ $\left[\begin{array}{lll}\delta \mathbf{p}^{\mathrm{T}} & c d t\end{array}\right]^{\mathrm{T}}$ and $c d t$ is the range error caused by the uncertainty of the receiver clock phase.

The normalized range and range rate difference equations are based on the computed values given by the inertial navigation module and measurements from the MF-GPS module and can be expressed as follows:

$$
\begin{aligned}
\mathbf{r}_{\mathrm{GNSS}}-\tilde{\mathbf{r}}_{\mathrm{INS}}= & -\mathbf{H}_{\mathrm{LOS}} \mathbf{C}_{\mathrm{n}}^{\mathrm{e}} \delta \mathbf{p}^{\mathrm{n}}+\mathbf{1} c d t+\boldsymbol{v}_{\mathrm{r}} \\
\dot{\mathbf{r}}_{\mathrm{GNSS}}-\tilde{\mathbf{r}}_{\mathrm{INS}}= & -\left(\mathbf{H}_{\mathrm{LOSV}} \mathbf{C}_{\mathrm{n}}^{\mathrm{e}}+\mathbf{H}_{\mathrm{LOS}} \mathbf{C}_{\mathrm{w}}^{\mathrm{e}} \mathbf{T}_{\mathrm{p}}^{\mathrm{v}}\right) \delta \mathbf{p}^{\mathrm{n}} \\
& -\mathbf{H}_{\mathrm{LOS}} \mathbf{C}_{\mathrm{w}}^{\mathrm{e}} \delta \mathbf{v}^{1}+\mathbf{1} c d \dot{t}+\boldsymbol{v}_{\mathrm{r}}
\end{aligned}
$$

where $\delta \mathbf{p}^{\mathrm{n}}=\left[\begin{array}{lll}\delta \varphi_{\mathrm{R}} & \delta \lambda_{\mathrm{R}} & \delta \mathrm{h}\end{array}\right]^{\mathrm{T}}$ is the linear position error and $\mathbf{C}_{\mathrm{n}}^{\mathrm{e}}$ is the transformation from the NEU frame to the ECEF frame and $c d \dot{t}$ is the range rate error caused by the uncertainty of the receiver clock frequency. $\mathbf{T}_{\mathrm{p}}^{\mathrm{v}}$ is a transformation matrix given by (41) in the appendix.

2) Normalized Position and Velocity Differences as Filter Observables: If the GPS-based navigation states are used to form the filter observables, the normalized position and velocity differences equations can be obtained as follows:

$$
\begin{aligned}
\left(\tilde{\varphi}_{\mathrm{INS}}-\tilde{\varphi}_{\mathrm{GNSS}}\right)\left(\mathrm{R}_{\mathrm{a}}+\mathrm{h}\right) & =\delta \varphi_{\mathrm{R}}+\nu_{\varphi-\mathrm{GNSS}} \\
\left(\tilde{\lambda}_{\mathrm{INS}}-\tilde{\lambda}_{\mathrm{GNSS}}\right)\left(\mathrm{R}_{\mathrm{a}}+\mathrm{h}\right) \cos (\varphi) & =\delta \lambda_{\mathrm{R}}+\nu_{\lambda-\mathrm{GNSS}} \\
\left(\tilde{\mathrm{h}}_{\mathrm{INS}}-\tilde{\mathrm{h}}_{\mathrm{GPS}}\right) & =\delta \mathrm{h}+\nu_{\mathrm{h}-\mathrm{GNSS}} \\
\tilde{\mathbf{v}}_{\mathrm{INS}}^{\mathrm{w}}-\mathbf{C}_{\mathrm{n}}^{\mathrm{w}} \tilde{\mathbf{v}}_{\mathrm{GNSS}}^{\mathrm{n}} & =\mathbf{T}_{\mathrm{p}}^{\mathrm{v}} \delta \mathbf{p}^{\mathrm{n}}+\delta \mathbf{v}^{1}+\boldsymbol{v}_{\mathbf{v}-\mathrm{GNSS}} .
\end{aligned}
$$

3) Normalized Attitude Difference Equation: If attitude information is available from the GPS attitude computation, the attitude difference equation can be normalized as follows:

$$
\text { Euler }_{\text {INS }}-\text { Euler }_{\text {GNSS }}=\mathbf{T}_{\text {tilt }}^{\text {Euler_err }} \partial \phi+\boldsymbol{v}_{\text {Att }}
$$

where Euler $_{\mathrm{GNSS}}$ are the Euler angles provided by GPS-based attitude determination, Euler $_{\text {INS }}$ are the Euler angles derived by the INS and $\boldsymbol{v}_{\text {Att }}$ is the attitude measurement noise.

If a magnetic heading sensor is available, the corresponding heading measurement is used:

$$
\begin{aligned}
\delta \psi_{\mathrm{w}}= & -\tan (\theta)\left[\cos \left(\psi_{\mathrm{w}}\right) \partial \phi_{\mathrm{x}}+\sin \left(\psi_{\mathrm{w}}\right) \partial \phi_{\mathrm{y}}\right] \\
& -\partial \phi_{\mathrm{z}}-b_{\mathrm{M}}+v_{\mathrm{M}}
\end{aligned}
$$

where $b_{\mathrm{M}}$ is the magnetic heading deviation and $v_{\mathrm{M}}$ is the measurement noise. $b_{\mathrm{M}}$ can be modeled as a combination of a random constant and a first-order Markov process. 
4) Normalized SRIMU Parity Residual Equation: For an SRIMU configuration, denoted by the design matrix $\mathbf{H}$, a parity matrix $\mathbf{P}$ can be constructed to satisfy the following constraints [28]:

$$
\mathbf{P H}=\mathbf{0} .
$$

By performing a linear transformation to the SRIMU measurement vector from the measurement space to the parity space, then

$$
\mathbf{p}=\mathbf{P m}=\mathbf{P} \delta_{\mathrm{D}}+\mathbf{P S} \mathbf{S}_{\mathrm{SF}} \mathbf{m} \mathbf{P G m}+\mathbf{P} \boldsymbol{v} .
$$

From (21), the above equation can be normalized as follows:

$$
\mathbf{p}=\mathbf{P}[\mathrm{I} \operatorname{Diag}(\mathbf{m}) \operatorname{Diag}(\Pi) \operatorname{Diag}(\Sigma)]\left[\begin{array}{c}
\delta_{\mathrm{D}} \\
\delta_{\mathrm{SF}} \\
\delta_{\mathrm{Az}} \\
\delta_{\mathrm{El}}
\end{array}\right]+\mathbf{P} \boldsymbol{v}
$$

where the parity residual equation is used as the filter observable.

Equations (15), (16), (18), and (21) constitute the dynamic model of the multi-mode navigation filter. Equations (24)-(29) and (32) provide the redundant measurement model of the multi-mode navigation filter. These equations and (21) can be configured for specific sensors. The reconfiguration is based on the operating modes given in Table I.

\section{SIMULATION STUDY AND RESULTS}

To evaluate the multi-mode navigation filter, a multisensor simulation model was developed, including an MF-GPS simulator and an SRIMU simulator. The MF-GPS simulator provides GPS-based attitude information and raw GPS measurements including pseudo-range and pseudo-range rate at the rate of $1 \mathrm{~Hz}$. The accuracy of the simulated GPS-based attitude estimates is $0.3^{\circ}(\sigma)$ if the GPS attitude solution is available. The SRIMU simulator can simulate several SRIMU configurations, including cube and cone configurations (for different cone-spin axis directions) and dodecahedron configurations. The SRIMU simulator, together with a true trajectory generator, generates realistic SRIMU measurements at the rate of $50 \mathrm{~Hz}$. The true trajectory is used as a reference to compute the error of the estimated aircraft motion states. The simulated gyro sensors have gyro drift rates from $1 \%$ h to $30^{\circ} / \mathrm{h}$. All simulated accelerometers have a typical bias error of $300 \mu \mathrm{g}$. The SRIMU simulator also simulates other error sources, including time-dependent sensor drifts, misalignments, scale factor errors and sensor noise. The simulation parameters of the inertial sensors used in the simulation are summarized in Table II. The simulated aircraft can perform arbitrary maneuvers

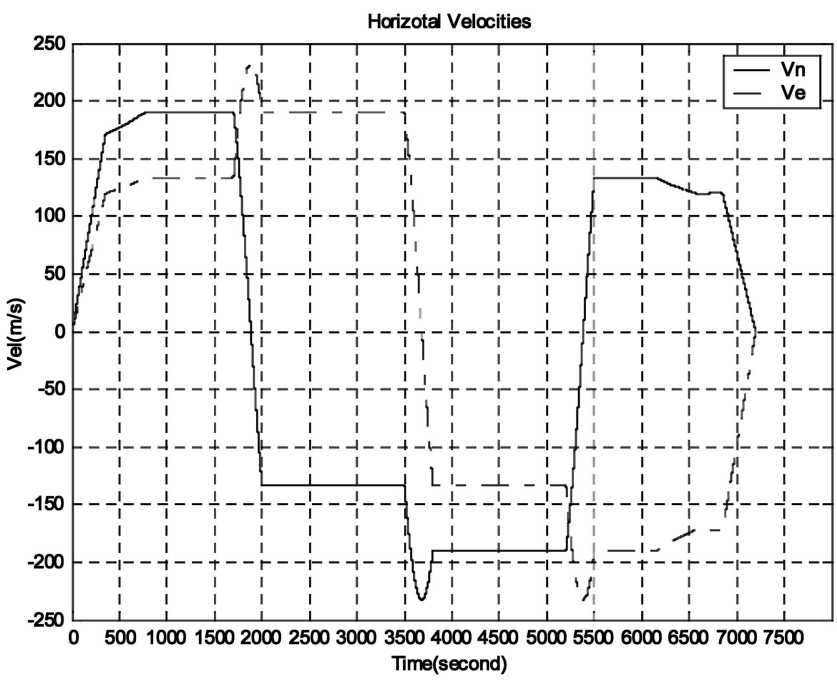

Fig. 4. Aircraft horizontal maneuvers.

to a maximum acceleration of $3.0 \mathrm{~g}$; Fig. 4 illustrates typical horizontal velocity maneuvers. The simulation results given here were based on a 5-sensor cone configuration with four grades of gyro sensors with gyro drift rates of $1 \% \mathrm{~h}, 10^{\circ} \mathrm{h}, 20^{\circ} \mathrm{h}$, and $30^{\circ} / \mathrm{h}$. In comparison with other 5-sensor configurations [26], the 5-sensor cone configuration is of interest because it exhibits the minimal performance degradation for one sensor failure, which is an important consideration in fault-tolerant design. In the following plotted results, the navigation state errors are the differences between the true and estimated values and the standard deviations are computed from the covariances of the navigation state errors estimated by the multi-mode filter.

Fig. 5 shows the navigation state errors given by the multi-mode navigation filter for SRIMU gyros with a drift rate of $1 \% \mathrm{~h}$. After the initial $10 \mathrm{~min}$ correction and alignment, the GPS attitude information was interrupted for 5 min every 5 min during a $2 \mathrm{~h}$ flight. The absolute attitude errors (the difference between the true and estimated values) remained within $0.15^{\circ}$. The lateral velocity errors were less than $0.5 \mathrm{~m} / \mathrm{s}$ with a probability of $98 \%$. Fig. 5 also indicates that the attitude estimates quickly converge to their stable state. Fig. 6 illustrates the convergence rates of the standard deviations of the navigation state errors estimated by the multi-mode filter. It can be seen that the standard deviations of the attitude errors gradually increase when GPS attitude information is no available and converge to $0.2^{\circ}$ after the initial 10 min period. Because an initial yaw error of $1.5^{\circ}$ was assumed, the convergence rate of the standard deviation of the yaw error is slower than the other attitude errors during the initial $10 \mathrm{~min}$ period of alignment. The standard deviations of the lateral velocity errors converged to $0.2^{\circ} \mathrm{m} / \mathrm{s}$. The vertical velocity exhibits a larger error compared with the lateral velocity error as a result of the vertical dilution of precision of GPS. 

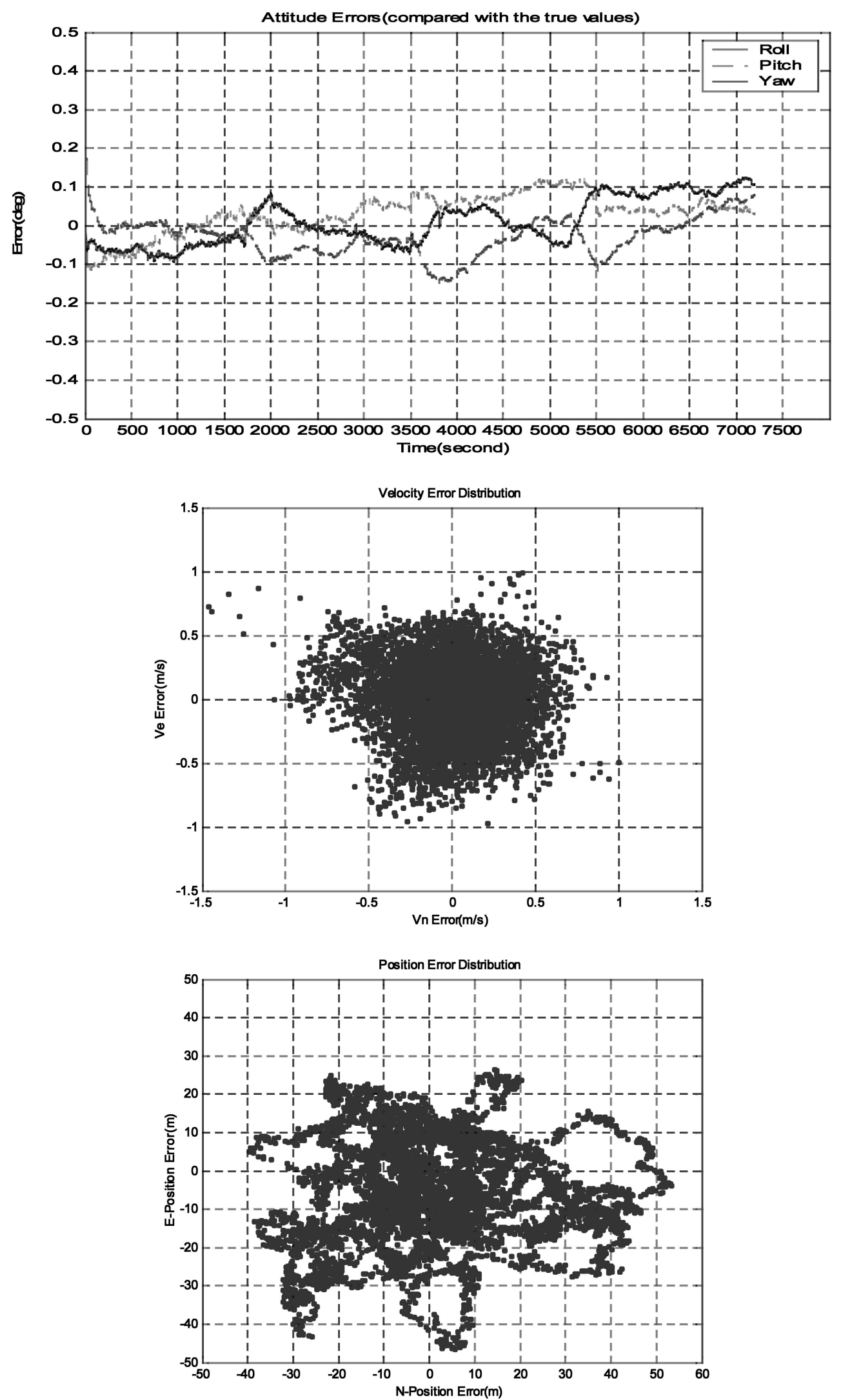

Fig. 5. Navigation state errors with Gyro drift of $1^{\circ} / \mathrm{h}$ and 5 min GPS attitude interruptions.

In Figs. 7 and 8, the GPS attitude information was interrupted for up to 12 min every 5 min after the initial 10 min alignment and correction, where
GPS raw measurements (pseudo-range and Doppler) were available. It can be observed from Figs. 7 and 8 that the convergence rate of the attitude estimates 

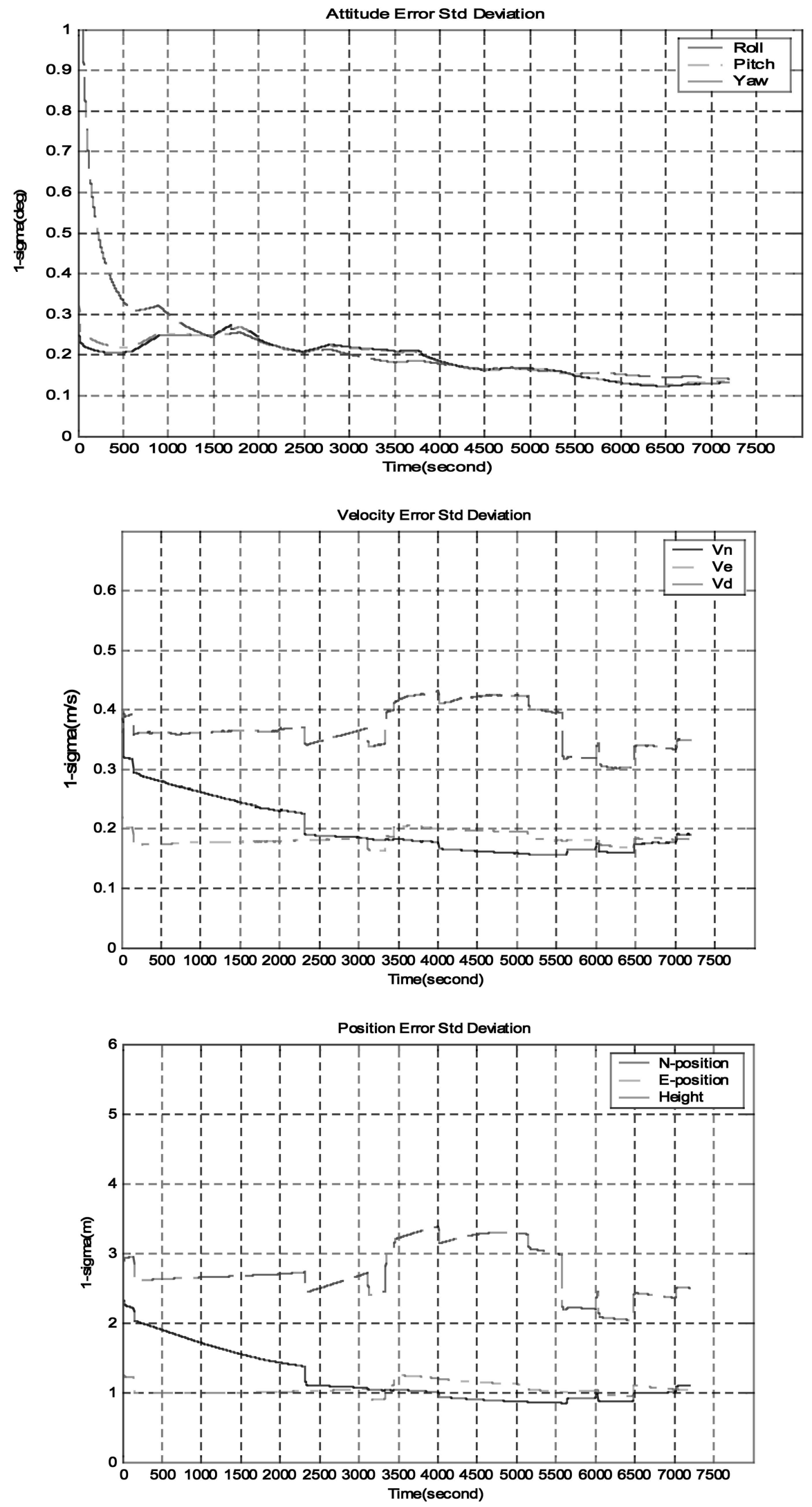

Fig. 6. Standard deviations with Gyro drift of $1^{\circ} / \mathrm{h}$ and 5 min GPS attitude interruptions.

is slightly slower and the accuracy of the velocity estimates is degraded in comparison with the previous 5 min interruption case. Nevertheless, the absolute attitude errors were kept within $0.2^{\circ}$. The reason is that, during the $5 \mathrm{~min}$ period of GPS attitude availability, there is not sufficient information for 

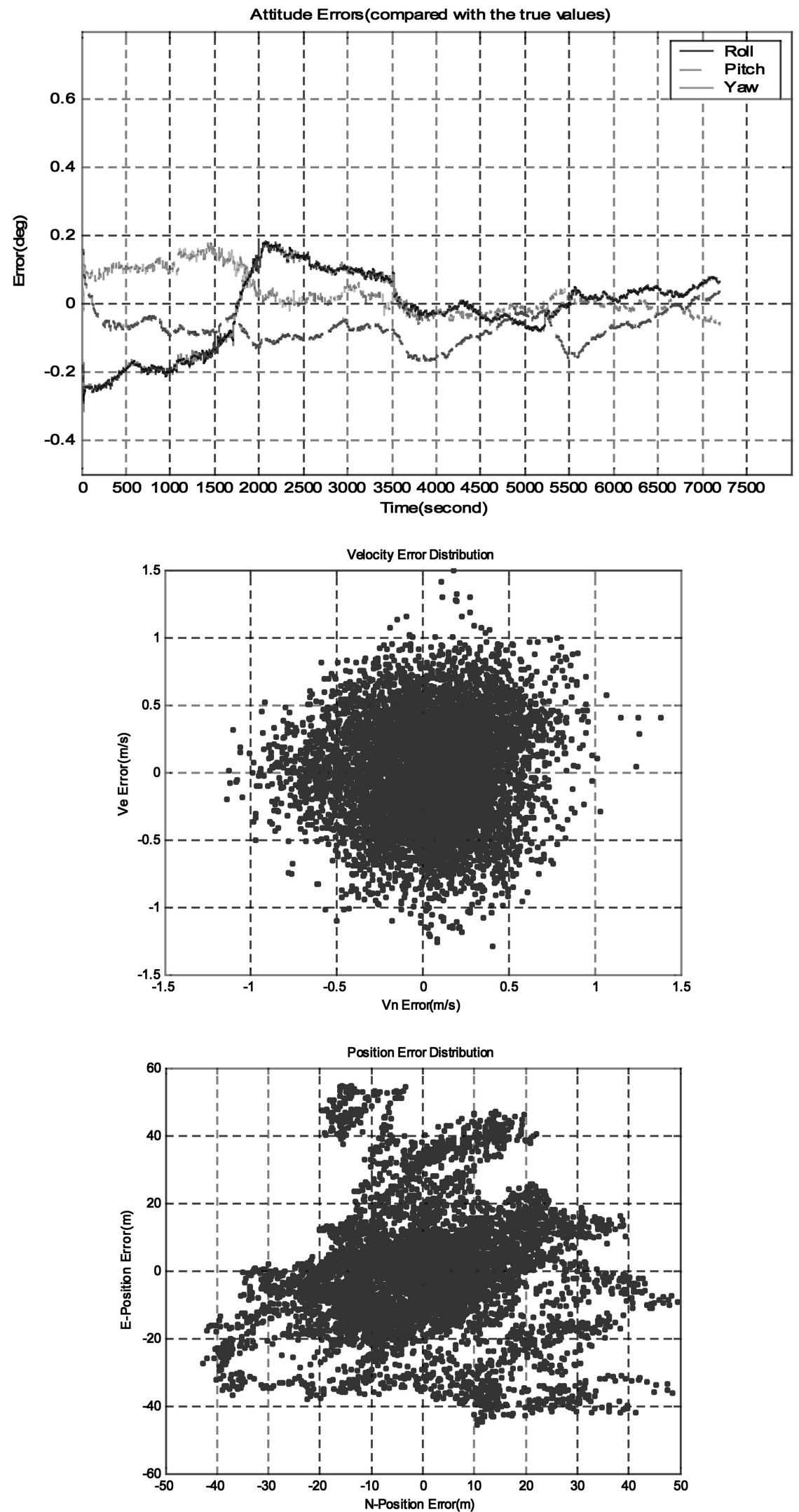

Fig. 7. Navigation state errors with Gyro drift of $1 \%$ h and 12 min GPS attitude interruptions.

in-flight correction of the gyro errors, following 12 min of GPS attitude interruption. It is also noted that significant attitude changes occurred during high dynamic maneuvers. In both cases, the sudden changes in standard deviations of the position and velocity error states were caused by the changes 

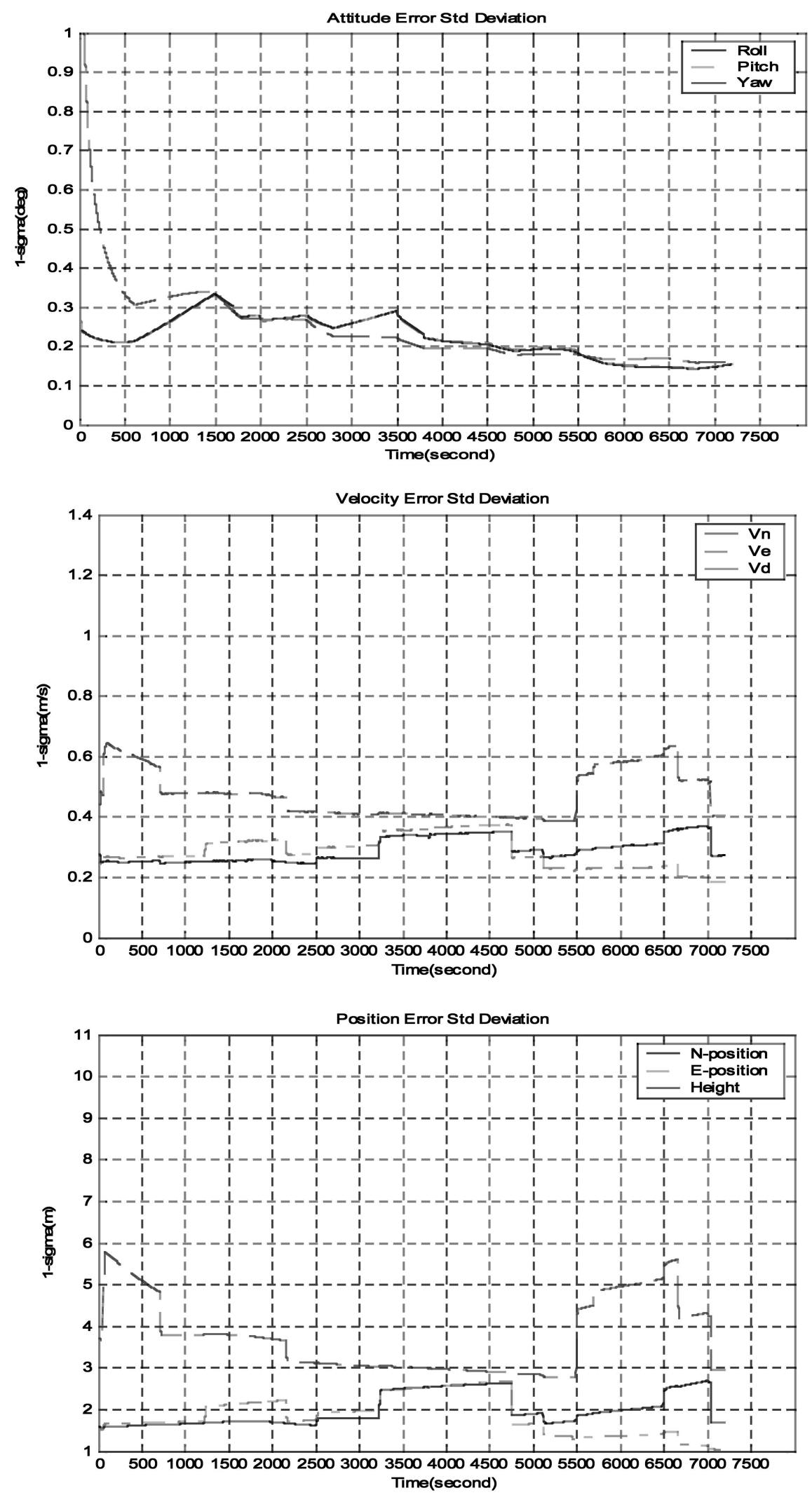

Fig. 8. Standard deviations with a Gyro drift of $1 \%$ h and $12 \min$ GPS attitude interruptions.

of the GPS satellite geometries. Fig. 8 shows that standard deviations of the lateral velocity errors of approximately $0.3 \mathrm{~m} / \mathrm{s}$, while the standard deviations of the attitude errors were greater than $0.2^{\circ}$. These simulation studies show that integration of an MF-GPS and an SRIMU with a gyro drift rate of $1 \% \mathrm{~h}$ 

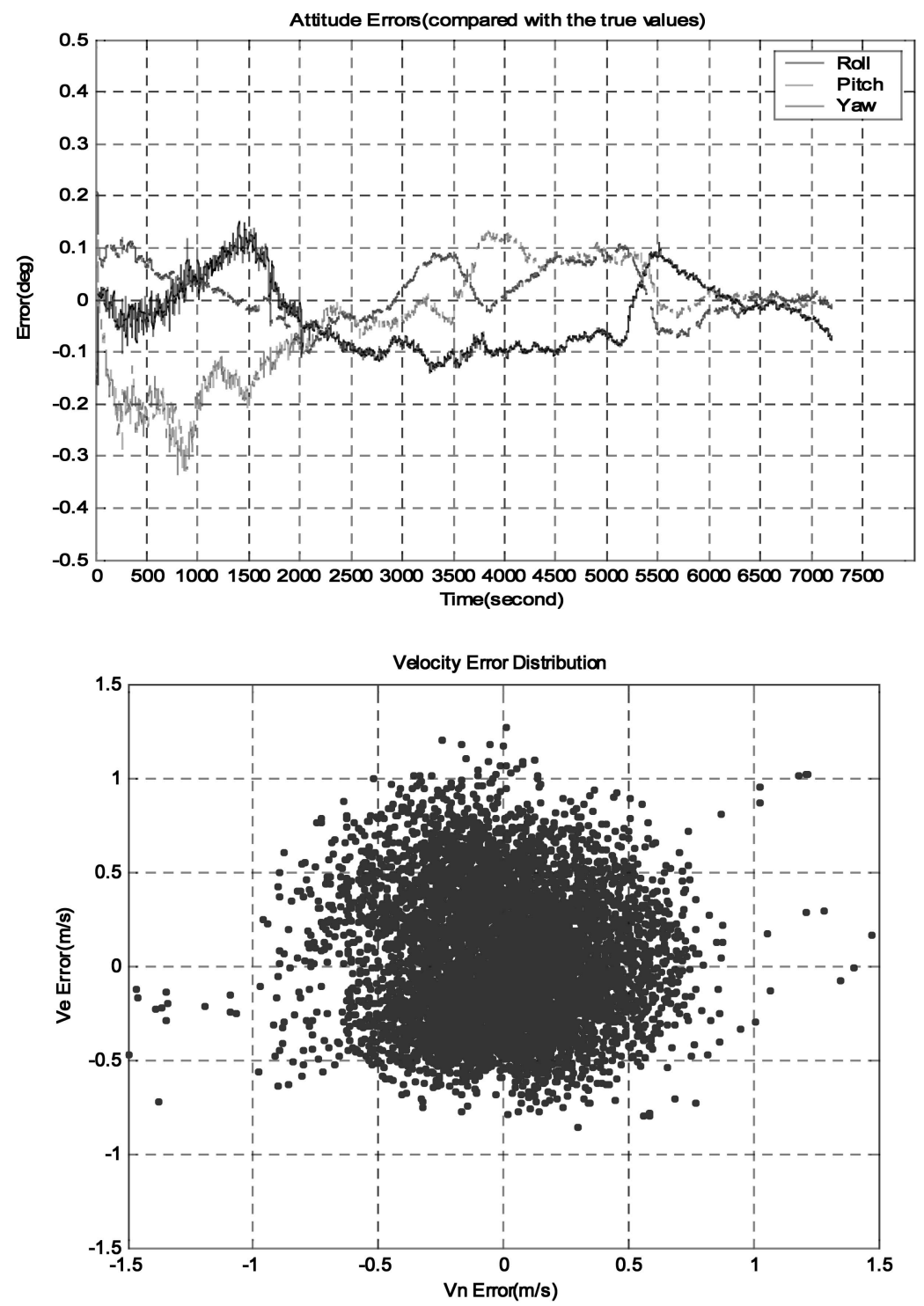

Fig. 9. Navigation state errors with a Gyro drift of $10^{\circ} / \mathrm{h}$ and 5 min GPS attitude interruptions.

can meet the navigation requirements of civil transport aircraft, if the loss of MF-GPS attitude information does not exceed $12 \mathrm{~min}$. The integrated navigation performance of the integrated navigation system is significantly improved when MF-GPS attitude information is available for a longer period, typically in excess of $5 \mathrm{~min}$.

Because the positioning accuracy of the multi-mode integrated navigation system is strongly influenced by the GPS positioning accuracy and the simulation results show that the accuracy of the position state estimation is not affected by the gyro drift error, the position error and standard deviation are omitted in the following simulation results.

In Figs. 9 and 10 the SRIMU gyro drift rate is increased from $1 \%$ h to $10^{\circ} \%$ h. After an initial $10 \mathrm{~min}$ alignment, the GPS attitude solution was again interrupted for $5 \mathrm{~min}$ every $5 \mathrm{~min}$. Although the convergence rate of the multi-mode navigation filter is slower, the integrated navigation system still exhibits satisfactory navigation performance if the period of interruption of the GPS attitude solution does not exceed $5 \mathrm{~min}$. If the initial alignment and correction time is increased from $10 \mathrm{~min}$ to $15 \mathrm{~min}$, the accuracy of the attitude estimates improves significantly. From Fig. 10, it can be seen that the convergence rate of the standard deviations of the attitude errors was much slower than the previous simulation results. Nevertheless, similar accuracy was achieved for the attitude and lateral velocity errors.

If the SRIMU gyro drift rate is increased to $20^{\circ} / \mathrm{h}$ and $30^{\circ} / \mathrm{h}$, the simulation studies show that the initial alignment time must also be increased to achieve sufficient attitude accuracy. The MF-GPS receiver is required to continuously provide the attitude solution for 5 min after an attitude interruption has taken place. Figs. 11 and 12 show the simulation results for SRIMU gyros with a drift rate of $20^{\circ} / \mathrm{h}$ where 

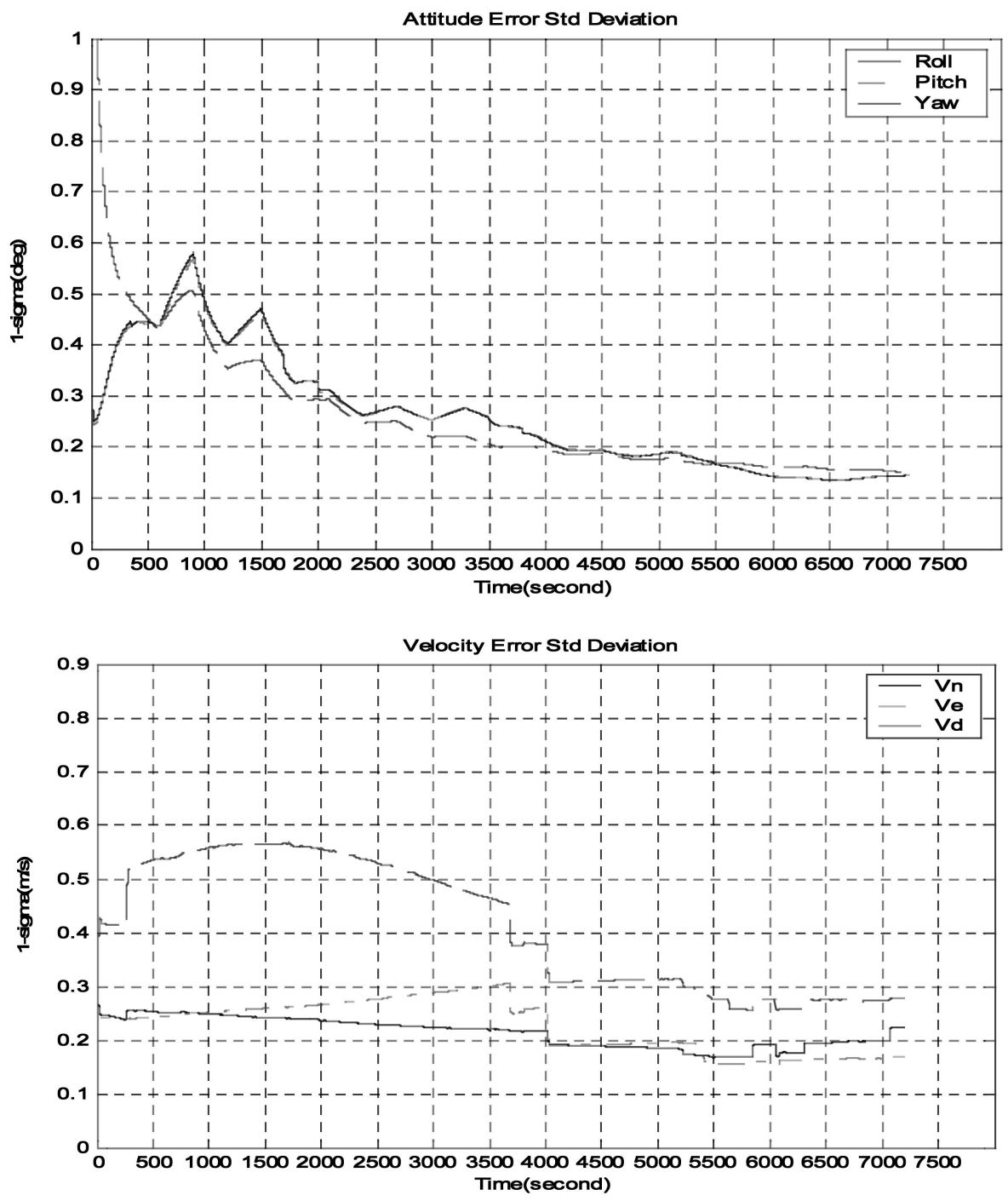

Fig. 10. Standard deviations with the Gyro drift of $10^{\circ} / \mathrm{h}$ and $5 \mathrm{~min}$ GPS attitude interruptions.

the GPS attitude information was interrupted for 5 min every 5 min after an initial alignment period of $15 \mathrm{~min}$.

Figs. 13 and 14 show the simulation results where the drift rate of the SRIMU gyros is $30 \%$. From Fig. 13, the attitude estimate errors converge to $0.2^{\circ}$ after $8 \mathrm{~min}$ and the accuracy of the velocity estimate is significantly degraded for high values of gyro drift rate. In order to develop lower cost inertial/MF-GPS integrated navigation systems, the MF-GPS receiver must be able to provide high rate and accurate Doppler estimates in order to increase the multi-mode filter update rate and to improve the accuracy of the velocity estimate. Current research on inertial-aiding GPS integration mechanisms offers a potential solution to this problem.

Further simulation studies were performed using GPS-based position and velocity information. Comparing the simulation results with values reported by Wolf [11], although the use of GPS-based position and velocity information can provide better estimates of the navigation states, the fusion has a significant disadvantage. If the number of visible satellites is less than four, then GPS-based position and velocity information will be unavailable and GPS attitude information alone cannot reduce the degradation of the navigation velocity state.

By observing all the error standard deviations plotted, it should be noted that the error covariances of the estimated states were slightly divergent when the multi-mode filter switched from GPS attitude modes to non-GPS attitude modes, especially where lower cost inertial sensors were modeled. However, the simulation results also show that this divergence can be controlled if the GPS attitude interruption does not exceed $5 \mathrm{~min}$ after the necessary initial alignment and calibration. The authors suggest that further work should be done in the stability analysis of the multi-mode filter.

In the simulation studies, the changes of the SRIMU configurations, which lead to reconfiguration of the parity residual equation, had no significant influence on the filter stability. 

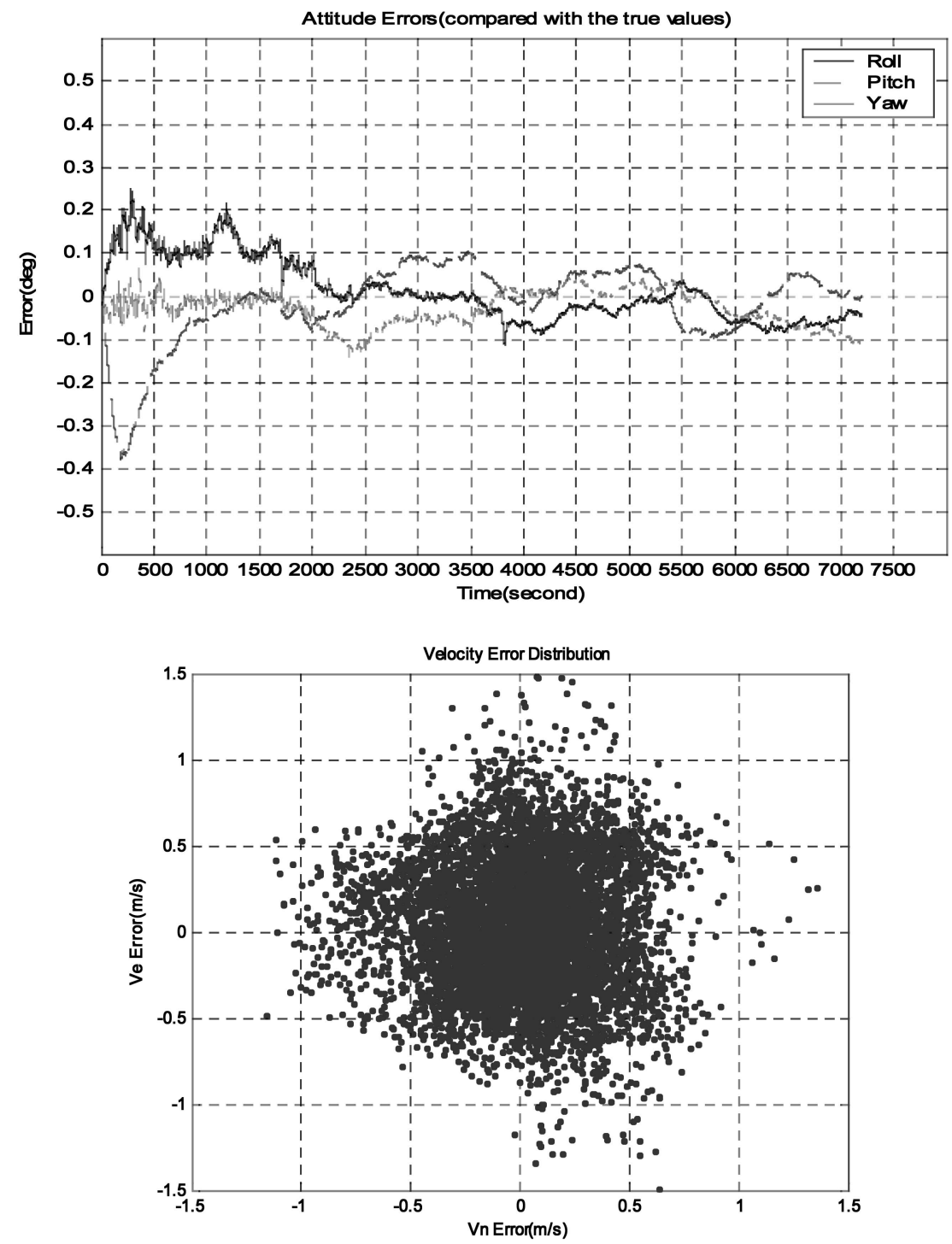

Fig. 11. Navigation state errors with Gyro drift of $20^{\circ} / \mathrm{h}$ and $5 \mathrm{~min}$ GPS attitude interruptions.

\section{CONCLUSIONS}

This simulation study has demonstrated the feasibility of integrating low-cost SRIMU systems with a multifunctional GPS receiver to achieve the required navigation performance using the multi-mode Kalman filter described in this paper. A wide range of low-cost inertial sensors were evaluated, covering the performance of the majority of currently available inertial sensors. For SRIMU gyro drift rates ranging from $1 \%$ to $10^{\circ} / \mathrm{h}$, the aiding GPS attitude information can be interrupted for up to $12 \mathrm{~min}$ (after an initial 10 min alignment). After a GPS attitude interruption occurs, the in-flight correction time is approximately $3 \mathrm{~min}$. In other words, the minimum requirement for the continuous availability of GPS attitude information is $3 \mathrm{~min}$. If the gyro drift rate value is increased from $20^{\circ} / \mathrm{h}$ to $30^{\circ} / \mathrm{h}$, the initial correction alignment time must be increased to 15 min (maximum) to achieve the required attitude accuracy. The minimum requirement for continuous availability of GPS attitude information is $5 \mathrm{~min}$ in order to correct SRIMU errors in flight. For larger values of gyro drift rate, the accuracy of the velocity estimate depends on the accuracy of GPS Doppler measurements. Compared with other methods, dynamic inertial error models were developed rather than simple state propagation models, which enabled sensor errors to be estimated by the multi-mode Kalman filter for low-cost SRIMUs. This simulation study shows that MF-GPS/SRIMU integrated systems can be used for general aviation aircraft and civil transport aircraft to provide control and navigation sensor systems.

APPENDIX

$$
\delta \mathbf{g}^{\mathrm{w}} \approx\left[\begin{array}{c}
0 \\
0 \\
-\frac{2 \mathrm{~g}_{0} \delta \mathrm{h}}{\mathrm{R}_{\mathrm{a}}+\mathrm{h}}
\end{array}\right]
$$



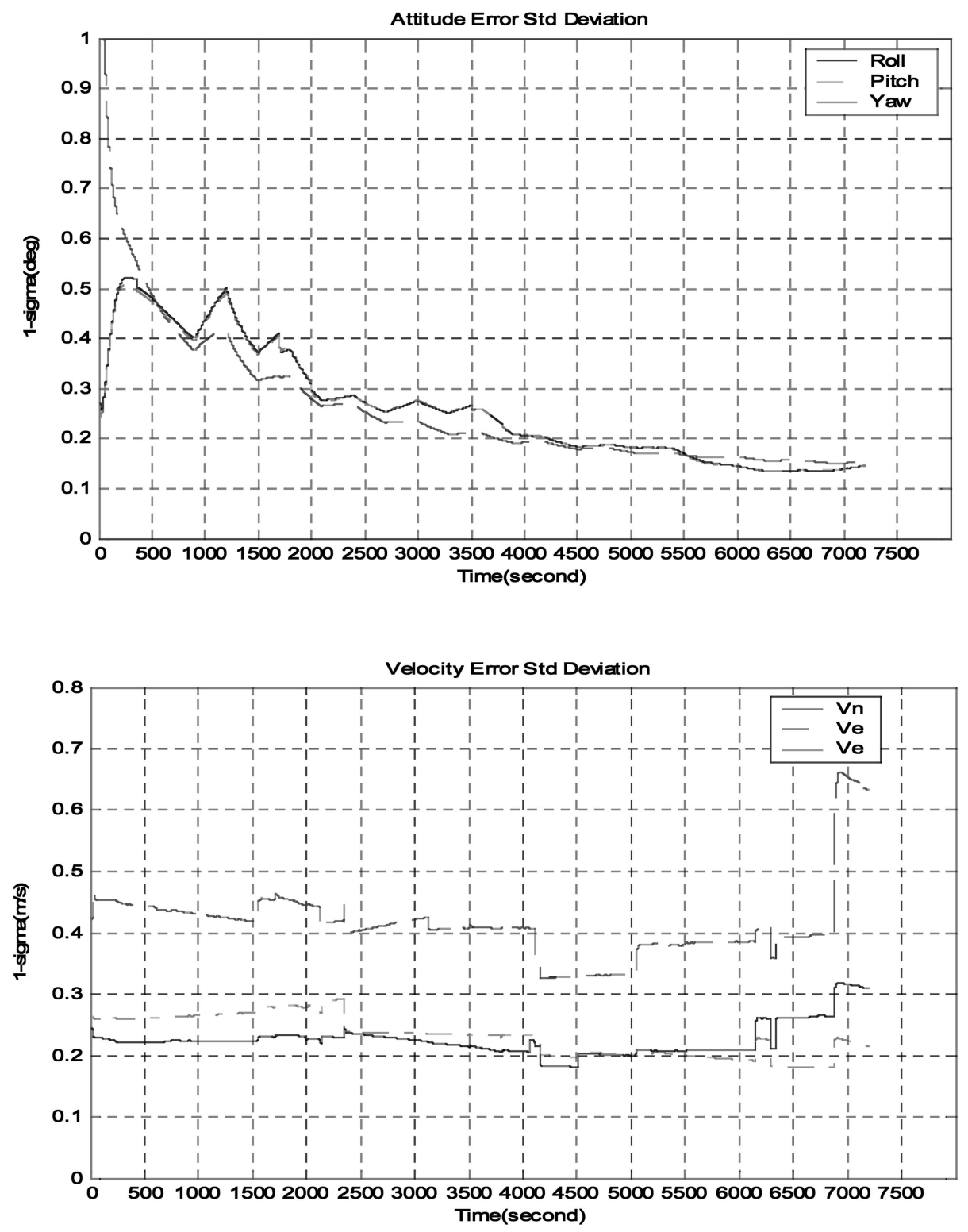

Fig. 12. Standard deviations with Gyro drift of $20^{\circ} / \mathrm{h}$ and $5 \mathrm{~min}$ GPS attitude interruptions.

$$
\begin{aligned}
& -\left(\mathbf{f}^{\mathbf{w}}+\mathbf{g}^{\mathrm{w}}\right) \times \delta \theta=\left[\begin{array}{ccc}
-\frac{\mathrm{f}_{\mathrm{z}}^{\mathrm{w}}+g_{0}}{\mathrm{R}_{\mathrm{a}}+\mathrm{h}} \cos (\alpha) & \frac{\mathrm{f}_{\mathrm{z}}^{\mathrm{w}}+g_{0}}{\mathrm{R}_{\mathrm{a}}+\mathrm{h}} \sin (\alpha) & 0 \\
-\frac{\mathrm{f}_{\mathrm{z}}^{\mathrm{w}}+g_{0}}{\mathrm{R}_{\mathrm{a}}+\mathrm{h}} \sin (\alpha) & -\frac{\mathrm{f}_{\mathrm{z}}^{\mathrm{w}}+g_{0}}{\mathrm{R}_{\mathrm{a}}+\mathrm{h}} \cos (\alpha) & 0 \\
\frac{\sin (\alpha) \mathrm{f}_{\mathrm{y}}^{\mathrm{w}}}{\mathrm{R}_{\mathrm{a}}+\mathrm{h}}+\frac{\cos (\alpha) \mathrm{f}_{\mathrm{x}}^{\mathrm{w}}}{\mathrm{R}_{\mathrm{a}}+\mathrm{h}} & \frac{\cos (\alpha) \mathrm{f}_{\mathrm{y}}^{\mathrm{w}}}{\mathrm{R}_{\mathrm{a}}+\mathrm{h}}-\frac{\sin (\alpha) \mathrm{f}_{\mathrm{x}}^{\mathrm{w}}}{\mathrm{R}_{\mathrm{a}}+\mathrm{h}} & 0
\end{array}\right]\left[\begin{array}{c}
\delta \varphi_{\mathrm{R}} \\
\delta \lambda_{\mathrm{R}} \\
\delta \mathrm{h}
\end{array}\right] \\
& \omega_{\mathrm{I} / \mathrm{e}}^{\mathrm{w}} \times \partial \theta=\left[\begin{array}{ccc}
\frac{\omega_{\mathrm{I} / \mathrm{e}, \mathrm{z}}^{\mathrm{w}} \cos (\alpha)}{\mathrm{R}_{\mathrm{a}}+\mathrm{h}} & -\frac{\omega_{\mathrm{I} / \mathrm{e}, \mathrm{z}}^{\mathrm{w}} \sin (\alpha)}{\mathrm{R}_{\mathrm{a}}+\mathrm{h}} & 0 \\
\frac{\omega_{\mathrm{I} / \mathrm{e}, \mathrm{z}}^{\mathrm{w}} \sin (\alpha)}{\mathrm{R}_{\mathrm{a}}+\mathrm{h}} & \frac{\omega_{\mathrm{I} / \mathrm{e}, \mathrm{z}}^{\mathrm{w}} \cos (\alpha)}{\mathrm{R}_{\mathrm{a}}+\mathrm{h}} & 0 \\
-\frac{\sin (\alpha) \omega_{\mathrm{I} / \mathrm{e}, \mathrm{y}}^{\mathrm{w}}-\frac{\cos (\alpha) \omega_{\mathrm{I} / \mathrm{e}, \mathrm{x}}^{\mathrm{w}}}{\mathrm{R}_{\mathrm{a}}+\mathrm{h}}}{\mathrm{R}_{\mathrm{a}}+\mathrm{h}} & -\frac{\cos (\alpha) \omega_{\mathrm{I} / \mathrm{e}, \mathrm{y}}^{\mathrm{w}}+\frac{\sin (\alpha) \omega_{\mathrm{I} / \mathrm{e}, \mathrm{x}}^{\mathrm{w}}}{\mathrm{R}_{\mathrm{a}}+\mathrm{h}}}{\mathrm{R}_{\mathrm{a}}+\mathrm{h}} & 0
\end{array}\right]\left[\begin{array}{c}
\delta \varphi_{\mathrm{R}} \\
\delta \lambda_{\mathrm{R}} \\
\delta \mathrm{h}
\end{array}\right]
\end{aligned}
$$



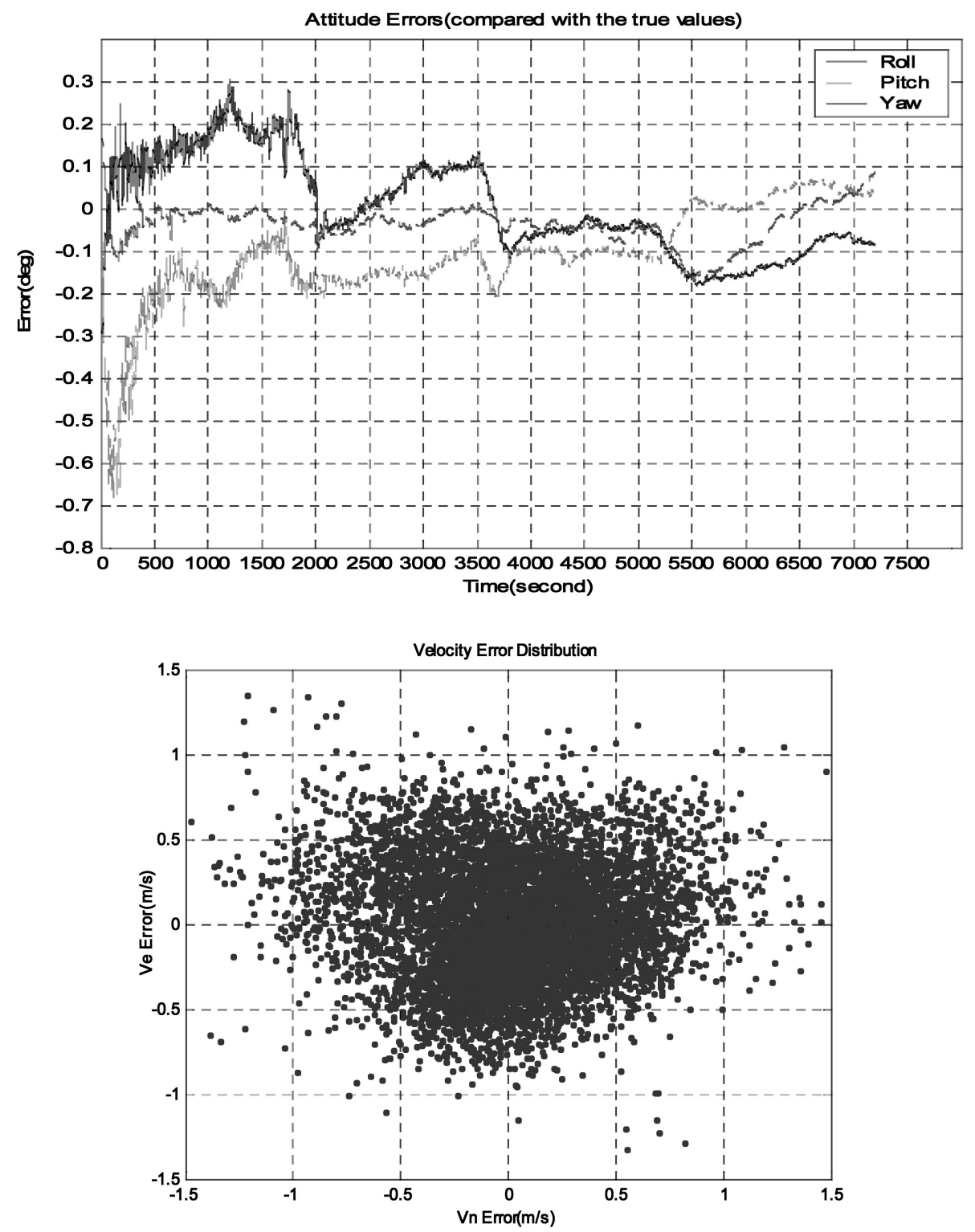

Fig. 13. Navigation state errors with Gyro drift of $30^{\circ} / \mathrm{h}$ and $5 \mathrm{~min}$ GPS attitude interruptions.

$$
\begin{aligned}
\delta \boldsymbol{\omega}_{\mathrm{e} / \mathrm{w}}^{\mathrm{w}}= & {\left[\begin{array}{ccc}
0 & \frac{1}{\mathrm{R}_{\mathrm{a}}+\mathrm{h}} & 0 \\
-\frac{1}{\mathrm{R}_{\mathrm{a}}+\mathrm{h}} & 0 & 0 \\
0 & 0 & 0
\end{array}\right]\left[\begin{array}{c}
\delta \mathrm{v}_{\mathrm{x}}^{1} \\
\delta \mathrm{v}_{\mathrm{y}}^{1} \\
\delta \mathrm{v}_{\mathrm{z}}^{1}
\end{array}\right]+\left[\begin{array}{ccc}
\frac{\sin (\alpha) \mathrm{v}_{\mathrm{z}}}{\left(\mathrm{R}_{\mathrm{a}}+\mathrm{h}\right)^{2}} & \frac{\cos (\alpha) \mathrm{v}_{\mathrm{z}}}{\left(\mathrm{R}_{\mathrm{a}}+\mathrm{h}\right)^{2}} & -\frac{\mathrm{v}_{\mathrm{y}}}{\left(\mathrm{R}_{\mathrm{a}}+\mathrm{h}\right)^{2}} \\
-\frac{\cos (\alpha) \mathrm{v}_{\mathrm{z}}}{\left(\mathrm{R}_{\mathrm{a}}+\mathrm{h}\right)^{2}} & \frac{\sin (\alpha) \mathrm{v}_{\mathrm{z}}}{\left(\mathrm{R}_{\mathrm{a}}+\mathrm{h}\right)^{2}} & \frac{\mathrm{v}_{\mathrm{x}}}{\left(\mathrm{R}_{\mathrm{a}}+\mathrm{h}\right)^{2}} \\
0 & 0 & 0
\end{array}\right]\left[\begin{array}{c}
\delta \varphi_{\mathrm{R}} \\
\delta \lambda_{\mathrm{R}} \\
\delta \mathrm{h}
\end{array}\right] } \\
\mathbf{T}_{\text {tilt }}^{\text {Euler_err }}= & {\left[\begin{array}{ccc}
-\frac{\cos \left(\psi_{\mathrm{w}}\right)}{\cos (\theta)} & -\frac{\sin \left(\psi_{\mathrm{w}}\right)}{\cos (\theta)} & 0 \\
\sin \left(\psi_{\mathrm{w}}\right) & -\cos \left(\psi_{\mathrm{w}}\right) & 0 \\
-\tan (\theta) \cos \left(\psi_{\mathrm{w}}\right) & -\tan (\theta) \sin \left(\psi_{\mathrm{w}}\right) & -1
\end{array}\right] } \\
\mathbf{T}_{\text {ang_err }}^{\text {geo_err }} & =\left[\begin{array}{ccc}
\sin (\alpha) & -\cos (\alpha) & 0 \\
\frac{\cos (\alpha)}{\cos (\varphi)} & \frac{\sin (\alpha)}{\cos (\varphi)} & 0 \\
-\tan (\varphi) \cos (\alpha) & -\tan (\varphi) \sin (\alpha) & -1
\end{array}\right]
\end{aligned}
$$



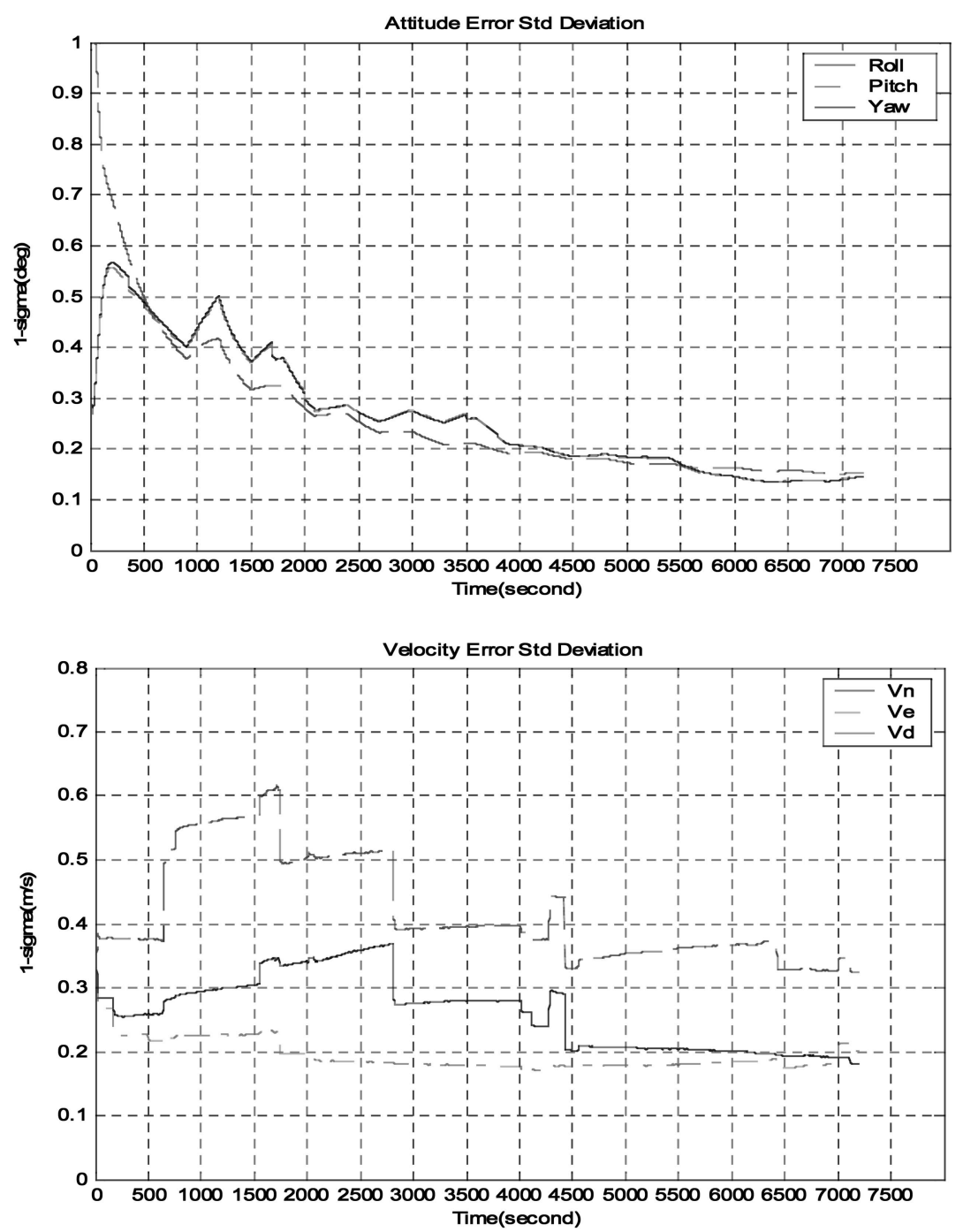

Fig. 14. Standard deviations with Gyro drift of $30^{\circ} / \mathrm{h}$ and $5 \mathrm{~min}$ GPS attitude interruptions.

$$
\begin{aligned}
\mathbf{H}_{\mathrm{GNSS}} & =\left[\begin{array}{cccc}
\frac{-\left(\mathrm{x}^{1}-\mathrm{x}_{0}\right)}{\rho_{0}^{1}} & \frac{-\left(\mathrm{y}^{1}-\mathrm{y}_{0}\right)}{\rho_{0}^{1}} & \frac{-\left(\mathrm{z}^{1}-\mathrm{z}_{0}\right)}{\rho_{0}^{1}} & 1 \\
\frac{-\left(\mathrm{x}^{2}-\mathrm{x}_{0}\right)}{\rho_{0}^{2}} & \frac{-\left(\mathrm{y}^{2}-\mathrm{y}_{0}\right)}{\rho_{0}^{2}} & \frac{-\left(\mathrm{z}^{2}-\mathrm{z}_{0}\right)}{\rho_{0}^{2}} & 1 \\
\frac{-\left(\mathrm{x}^{3}-\mathrm{x}_{0}\right)}{\rho_{0}^{3}} & \frac{-\left(\mathrm{y}^{3}-\mathrm{y}_{0}\right)}{\rho_{0}^{3}} & \frac{-\left(\mathrm{z}^{3}-\mathrm{z}_{0}\right)}{\rho_{0}^{3}} & 1 \\
\vdots & \vdots & \vdots & \vdots \\
\frac{-\left(\mathrm{x}^{m}-\mathrm{x}_{0}\right)}{\rho_{0}^{m}} & \frac{-\left(\mathrm{y}^{m}-\mathrm{y}_{0}\right)}{\rho_{0}^{m}} & \frac{-\left(\mathrm{z}^{m}-\mathrm{z}_{0}\right)}{\rho_{0}^{m}} & 1
\end{array}\right] \\
= & {\left[\begin{array}{llll}
\mathbf{H}_{\mathrm{LOS}} \mathbf{1}
\end{array}\right] }
\end{aligned}
$$$$
\mathbf{H}_{\mathrm{LOSV}}=\left[\begin{array}{ccc}
\frac{-\left(\dot{\mathrm{x}}^{1}-\dot{\mathrm{x}}_{0}\right)}{\rho_{0}^{1}} & \frac{-\left(\dot{\mathrm{y}}^{1}-\dot{\mathrm{y}}_{0}\right)}{\rho_{0}^{1}} & \frac{-\left(\dot{\mathrm{z}}^{1}-\dot{\mathrm{z}}_{0}\right)}{\rho_{0}^{1}} \\
\frac{-\left(\dot{\mathrm{x}}^{2}-\dot{\mathrm{x}}_{0}\right)}{\rho_{0}^{2}} & \frac{-\left(\dot{\mathrm{y}}^{2}-\dot{\mathrm{y}}_{0}\right)}{\rho_{0}^{2}} & \frac{-\left(\dot{\mathrm{z}}^{2}-\dot{\mathrm{z}}_{0}\right)}{\rho_{0}^{2}} \\
\frac{-\left(\dot{\mathrm{x}}^{3}-\dot{\mathrm{x}}_{0}\right)}{\rho_{0}^{3}} & \frac{-\left(\dot{\mathrm{y}}^{3}-\dot{\mathrm{y}}_{0}\right)}{\rho_{0}^{3}} & \frac{-\left(\dot{\mathrm{z}}^{3}-\dot{\mathrm{z}}_{0}\right)}{\rho_{0}^{3}} \\
\vdots & \vdots & \vdots \\
\frac{-\left(\dot{\mathrm{x}}^{m}-\dot{\mathrm{x}}_{0}\right)}{\rho_{0}^{m}} & \frac{-\left(\dot{\mathrm{y}}^{m}-\dot{\mathrm{y}}_{0}\right)}{\rho_{0}^{m}} & \frac{-\left(\dot{\mathrm{z}}^{m}-\dot{\mathrm{z}}_{0}\right)}{\rho_{0}^{m}}
\end{array}\right]
$$

$$
\mathbf{T}_{\mathrm{p}}^{\mathrm{v}}=\left[\begin{array}{ccc}
\frac{\mathrm{v}_{\mathrm{z}}}{\mathrm{R}_{\mathrm{a}}+\mathrm{h}} \cos (\alpha) & -\frac{\mathrm{v}_{\mathrm{z}}}{\mathrm{R}_{\mathrm{a}}+\mathrm{h}} \sin (\alpha) & 0 \\
\frac{\mathrm{v}_{\mathrm{z}}}{\mathrm{R}_{\mathrm{a}}+\mathrm{h}} \sin (\alpha) & \frac{\mathrm{v}_{\mathrm{z}}}{\mathrm{R}_{\mathrm{a}}+\mathrm{h}} \cos (\alpha) & 0 \\
-\left(\frac{\mathrm{v}_{\mathrm{x}}}{\mathrm{R}_{\mathrm{a}}+\mathrm{h}} \cos (\alpha)+\frac{\mathrm{v}_{\mathrm{y}}}{\mathrm{R}_{\mathrm{a}}+\mathrm{h}} \sin (\alpha)\right) & \left(\frac{\mathrm{v}_{\mathrm{x}}}{\mathrm{R}_{\mathrm{a}}+\mathrm{h}} \sin (\alpha)-\frac{\mathrm{v}_{\mathrm{y}}}{\mathrm{R}_{\mathrm{a}}+\mathrm{h}} \cos (\alpha)\right) & 0
\end{array}\right]
$$




\section{REFERENCES}

[1] Allerton, D. J., and Jia, H.

A review of multisensor fusion methodologies for aircraft navigation systems.

The Journal of Navigation, 58, 3 (Sept. 2005), 405-417.

[2] Ebner, R. E., and Klein, A. D.

Integrated navigation/flight control for future high performance aircraft.

AGARD-AG-314, Analysis, Design and Synthesis

Methods for Guidance and Control Systems, 1995, V1-8.

[3] Schmidt, G. T.

GPS/INS technology trends for military systems.

Draper Technology Digest, (1998), 2-13.

http://www.draper.com/publications/digest98/paper13.pdf.

[4] Alban, S., Akos, D. M., Rock, S. M., and

Gebre-Egziabher, D.

Performance analysis and architectures for INS-aided

GPS tracking loops.

Proceedings of ION National Technical Meeting, Jan.

2003. http://pei2005.free.fr/backup/documents/documents_ externes/GPSINS/AlbanIONNTM03.pdf.

[5] Van Graas, F., and Braasch, M. S.

GPS interferometric attitude and heading determination:

Flight test results.

Navigation, 41 (Fall 1991), 297-316.

[6] Cohen, C. E., Parkinson, B. W., and McNally, B. D.

Flight tests of attitude determination using GPS compared against an inertial navigation unit.

Navigation, 41, 1 (Spring 1994).

[7] Hayward, R. C., Gebre-Egziabher, D., and Powell, J. D.

GPS-based attitude for aircraft.

Proceedings of 5th Saint Petersburg International

Conference on Integrated Navigation System, May 1998.

waas.stanford.edu/ wwu/papers/gps/PDF/att_for_aircraft_ rch1998.pdf.

[8] Crassidis, J. L., Markley, F. L., and Lightsey, E. G. Global positioning system integer ambiguity resolution without attitude knowledge.

AIAA Journal of Guidance, Control, and Dynamics, 22, 2 (Mar.-Apr. 1999), 212-218.

[9] Lightsey, E. G., Crassidis, J. L., and Markley, F. L. Fast integer ambiguity resolution for GPS attitude determination.

Proceedings of AIAA Guidance, Navigation, and Control Conference, 1999, AIAA-99-3967, 1999. www.acsu.buffalo.edu/ johnc/gps_gnc99.pdf.

[10] Yang, Y. C., and Farrell, J. A.

Fast ambiguity resolution for GPS/IMU attitude determination.

Proceedings of ION GPS-01, 2001.

[11] Wolf, R., Hein, G., Eissfeller, B., and Loehnert, E. An integrated low cost GPS/INS attitude determination and position location system.

Proceedings of ION GPS-96, 1996, 975-981. http://www.ifen.com/.

[12] Gebre-Egziabher, D., Hayward, R. C., and Powell, J. D. A low-cost GPS/inertial attitude heading reference system (AHRS) for general aviation applications.

Presented at IEEE Position, Location, and Navigation Symposium, Apr. 1998. waas.stanford.edu/ wwu/papers/ gps/PDF/cheap_ahrs_4_ga_dge98.pdf.

[13] Buchler, R. J., Lee, C. A., and Brockstein, A. J.

Design and test of a synergistic interferometric GPS-INS. Proceedings of IEEE Position, Location, and Navigation Symposium, Apr. 1996, 612-619.
[14] Crassidis, J. L., and Lightsey, E. G.

Attitude determination using combined gps and three-axis magnetometer data.

Proceedings of International Workshop on Applications of the Global Positioning System, Feb. 2000, 522-534. www.acsu.buffalo.edu/ johnc/gps_brek.pdf.

[15] Daly, K. C., Gai, E., and Harrison, J. V.

Generalized likelihood ratio test for FDI in redundant sensor configurations.

Journal of Guidance Control, 2, 1 (Jan. 1979), 9-17.

[16] Hall, S. R., Motyka, P., Gai, E., and Deyst, J. J., Jr.

In-flight parity vector compensation for FDI.

IEEE Transactions on Aerospace and Electronics Systems,

AES-19, 5 (Sept. 1983), 668-675.

[17] Allerton, D. J., and Jia, H.

An error compensation method for skewed redundant inertial configurations.

Proceedings of ION 58th Annual Meeting/GIGTF 21st

Guidant Test Symposium, June 24-26, 2002, 142-147.

[18] Diggelen, F., and Brown, A. K.

Mathematical aspects of GPS RAIM.

Proceedings of IEEE Position, Location, and Navigation Symposium, 1994, 733-738.

[19] Zink, T., Eissfeller, B., Lohnert, E., and Wolf, R.

Analyses of integrity monitoring techniques for a global navigation satellite system (GNSS-2).

Proceedings of IAIN-ION Annual Technical Meeting, 2000. www.ifen.com/publications/IAIN_ION_2000_Paper.pdf.

[20] Hewitson, S.

GNSS receiver autonomous integrity monitoring: A

separability analysis.

Proceedings of ION GPS-03, 2003.

[21] Brenner, M.

Integrated GPS/inertial fault detection availability.

Proceedings of ION GPS-95, 1995.

[22] Hanlon, P. D., and Maybeck, P. S.

Characterization of Kalman filter residuals in the presence of mismodeling.

IEEE Transactions on Aerospace and Electronic Systems, 36, 1 (Jan. 2000), 114-131.

[23] Hanlon, P. D., and Maybeck, P. S.

Multiple-model adaptive estimation using a residual correlation Kalman filter bank.

IEEE Transactions on Aerospace and Electronic Systems, 36, 2 (Apr. 2000), 393-406.

[24] Maybeck, P. S.

Stochastic Models, Estimation, and Control, Vol. 1.

New York: Academic Press, 1979.

[25] Roger, R. M.

Applied Mathematics in Integrated Navigation Systems. New York: AIAA, 2000.

[26] Jia, H.

Data fusion methodologies for multisensor aircraft navigation systems.

Ph.D. dissertation, Cranfield University, Bedfordshire, UK, 2004.

[27] IEEE Standard Specification Format Guide and Test Procedure for Single-Axis Interferometric Fiber Optic Gyros.

IEEE Std 952-1997.

[28] Brown, A., and Sturza, M. A.

The effect of geometry on integrity monitoring performance.

Proceedings of the ION 46th Annual Meeting, June 26-28, 1990.

[29] Soloviev, A., and van Graas, F.

Review of potential applications of low-cost GPS/INS for general aviation.

Proceedings of ION National Technical Meeting, Jan. 2003. 


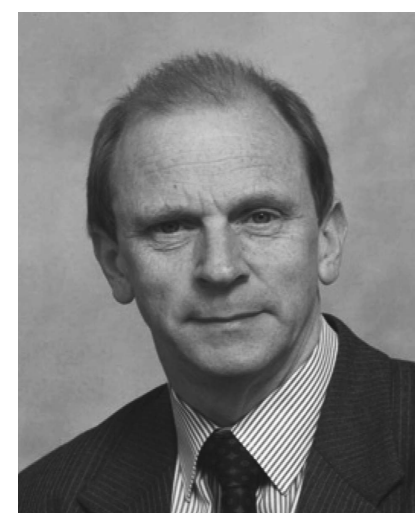

D. J. Allerton was born in Portsmouth in the UK in 1949. He obtained a degree in computer systems engineering from Rugby College of Engineering Technology in 1972 and a Ph.D. in control engineering from the University of Cambridge in 1977.

He worked from GEC Marconi as a principal systems engineer from 1977 to 1981 on real-time embedded systems. He moved to the Department of Electronics and Computer Science as a lecturer in 1981 and was appointed to a Senior Lectureship in 1987. He was appointed to the GEC Professorship in Avionics in the College of Aeronautics at Cranfield University in 1991 and moved to the Department of Automatic Control and Systems Engineering at the University of Sheffield in 2003, where he is the Professor of Computer Systems Engineering. He has published widely in the areas of computer architecture, VLSI design, real-time simulation and navigation systems. His major area of research covers real-time flight simulation, computer graphics and distributed computing.

Dr. Allerton is a fellow of the Institution of Electrical Engineers and a fellow of the Royal Aeronautical Society. He has served on the UK Foresight Defence and Aerospace Panel and is currently a member of the UK National Advisory Committee for Avionics and also the Avionics Committee of the Royal Aeronautical Society.

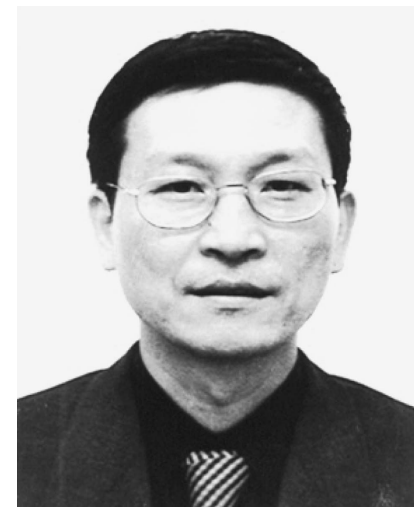

Huamin Jia was born in Xian in P.R. China in 1959. He obtained an M.Sc. (Eng.) in computer software engineering from the University of Science and Technology of China in 1988, and a Ph.D. in avionics systems from Cranfield University, UK, in 2004.

Dr. Jia worked in China from 1982 to 1996 and in the USA from 1996 to 2000 on the design of integrated navigation systems and development of navigation and control algorithms and software systems. He moved to the College of Aeronautics at Cranfield University in 2000 as a research assistant to study for his $\mathrm{Ph} . \mathrm{D}$. in avionics systems. He is now a lecturer of avionics systems at Cranfield University. His research interests cover integrated navigation systems, multisensor data fusion for aerial vehicle guidance, navigation and control, multi-agent formation, and avionics system safety assessments. 